\title{
The Fault Feature Extraction of Rolling Bearing Based on EMD and Difference Spectrum of Singular Value
}

\author{
Te Han, Dongxiang Jiang, and Nanfei Wang \\ State Key Lab of Control and Simulation of Power System and Generation Equipment, Department of Thermal Engineering, \\ Tsinghua University, Beijing 100084, China
}

Correspondence should be addressed to Te Han; hant15@mails.tsinghua.edu.cn

Received 3 December 2015; Revised 1 March 2016; Accepted 15 March 2016

Academic Editor: Peng Chen

Copyright (C) 2016 Te Han et al. This is an open access article distributed under the Creative Commons Attribution License, which permits unrestricted use, distribution, and reproduction in any medium, provided the original work is properly cited.

\begin{abstract}
Nowadays, the fault diagnosis of rolling bearing in aeroengines is based on the vibration signal measured on casing, instead of bearing block. However, the vibration signal of the bearing is often covered by a series of complex components caused by other structures (rotor, gears). Therefore, when bearings cause failure, it is still not certain that the fault feature can be extracted from the vibration signal on casing. In order to solve this problem, a novel fault feature extraction method for rolling bearing based on empirical mode decomposition (EMD) and the difference spectrum of singular value is proposed in this paper. Firstly, the vibration signal is decomposed by EMD. Next, the difference spectrum of singular value method is applied. The study finds that each peak on the difference spectrum corresponds to each component in the original signal. According to the peaks on the difference spectrum, the component signal of the bearing fault can be reconstructed. To validate the proposed method, the bearing fault data collected on the casing are analyzed. The results indicate that the proposed rolling bearing diagnosis method can accurately extract the fault feature that is submerged in other component signals and noise.
\end{abstract}

\section{Introduction}

Rolling bearings are the most important components in aeroengines that support the rotor system. The working environment is extremely harsh. Because of the high speed, the complex stress situation, and the high temperature, rolling bearing failure occurs frequently. Therefore, it is significant to extract the fault features, which judge the state of bearing in time. Normally, the vibration signal is measured from the bearing chock. However, only the limited sensors that are installed on aeroengine casing can be used. The vibration signal of the fault bearing is attenuated in the process of being transmitted to the casing. Additionally, there are other structural vibrations and noise on casing. The bearing fault information is easily masked, making it difficult to detect the fault feature of bearing at an early stage. Today, it is still not clear that the bearing fault diagnosis based on casing sensors is valid [1]. Therefore, it is challenging to propose an effective signal processing method for the fault diagnosis of rolling bearings based on the vibration signal measured on casing.
When faults occur in a rolling bearing, the impact will cause a resonance of the whole system. The vibration signal always shows the feature of modulation. Hence, the demodulated resonance technique has been widely used in this field [2-5]. This technique consists of two conventional steps, the bandpass filtering and the Hilbert-transform. Then the fault characteristic frequency of bearing can be detected. However, the selection of central frequency and bandwidth, which directly influences the accuracy of analysis results, always depends on experience. To solve this problem, empirical mode decomposition (EMD), which is an adaptive decomposition method, is put forward [6]. This method is based on the local characteristic time scale of the signal and decomposes the signal, which contains different components, into a series of intrinsic mode functions called IMFs. Each IMF can be an amplitude-component and frequency modulation (AM-FM) signal. Therefore, the fault signal of the bearing consists of a certain IMF. By applying envelope demodulation to the IMF, the fault feature frequency of bearing can be extracted. Recently, many improved adaptive decomposition 
methods based on EMD such as ensemble empirical mode decomposition (EEMD), local mean decomposition (LMD), and variational mode decomposition (VMD) are proposed [7-10]. However, all of these methods fail to extract the fault feature when the signal has strong noise and multiple components. Therefore, the further research related to algorithms that search for the informative frequency band of each IMF is needed [11-13].

In the fault diagnosis field of the rolling bearing, the research about singular value decomposition (SVD) receives more attention. As a nonlinear filtering technique, it decomposes the Hankel matrix containing the signal characteristics into the signal subspaces represented by singular values. The smaller singular values should be removed to achieve noise reduction and the signal can be reconstructed by the front singular values. SVD has excellent performance in the noiseremoval and fault feature extraction of rolling bearing [1417]. In some research, singular values are also served as fault feature parameters. The results show that this method has high accuracy for rolling bearing fault diagnosis [1820]. Nevertheless, the effect of the research above relies on the selection of effective singular values [21, 22]. Zhao et al. proposed the concept of the difference spectrum of singular value and noted that the position of the maximal peak in the difference spectrum can be used to determine the number of useful singular values $[23,24]$. The amount of research about the difference spectrum of singular value is small so far. In this paper, the component signals can be reflected by the peaks on the difference spectrum when original signal contains uncorrelated components. Whether the peak is prominent depends on the energy of the signal it represents. When bearing fails at an early stage or other component signals are strong, the peak corresponding to the fault signal of bearing will be weak in the difference spectrum. Therefore, this paper uses EMD as a kind of signal preprocessing method. Firstly, the vibration signal of rolling bearing is decomposed into a series of IMFs by using EMD. The resonance signal caused by rolling bearing fault is always of high frequency. Hence, the IMFs representing high frequency information are selected. Secondly, selected IMFs are processed by SVD. The difference spectrum of singular value can be obtained. The component signals are reconstructed according to the peaks of difference spectrum. If there are several peaks on the spectrum, the correlated kurtosis value can be utilized for selection criterion from these components. Finally, Hilbert envelope demodulation and spectral analysis of the reconstructed signal are applied.

The remaining part of this paper is organized as follows. In Section 2, the EMD and SVD algorithm are introduced firstly. Secondly, the structure of Hankel matrix and the difference spectrum of singular value are studied. Finally, the correlated kurtosis method is introduced to select effective peaks on the difference spectrum. In Section 3, through digital signal simulation analysis, the rolling bearing fault feature extraction strategy based on EMD and the difference spectrum are illustrated. Then, Section 4.1 presents the bearing fault experiment that simulates three fault conditions with different crack widths on outer ring. The vibration data measured from the bearing block and casing are processed by envelope demodulation method, respectively, which is widely used in engineering. The analysis results show that the fault characteristic frequency from the data collected on the casing cannot be extracted when the crack is slight. To validate the proposed method, the bearing fault data measured on the casing are used in two case studies in Section 4.2. The results show that the proposed method can accurately extract the fault feature that is submerged in other components of the signal and noise. Finally, the conclusions are listed in Section 5.

\section{Methods}

2.1. Empirical Mode Decomposition (EMD). In essence, EMD can separate the movements or trends of the signal in different scales step by step and produce a series of data sequences in different characteristic scales. Each sequence is called an intrinsic mode function (IMF). The IMFs are different and can change with the variation of the signal. Therefore, EMD is a self-adaptive signal decomposition method. The IMFs include different frequency bands ranging from being high to low. Usually, the most striking and important information of the original signal is concentrated in lower-order IMFs. The detailed decomposition process of EMD is explained in the literature [6].

2.2. Singular Value Decomposition (SVD) Principle. The SVD is defined as follows: for a matrix $\mathbf{A} \in \mathbf{R}^{m \times n}$, there are two orthogonal matrices $\mathbf{U} \in \mathbf{R}^{m \times m}$ and $\mathbf{V} \in \mathbf{R}^{n \times n}$, and the formula below is established:

$$
\mathbf{A}=\mathbf{U} \boldsymbol{\Sigma} \mathbf{V}
$$

where $\boldsymbol{\Sigma}$ is a diagonal matrix, $\boldsymbol{\Sigma}=\left[\operatorname{diag}\left(\sigma_{1}, \sigma_{2}, \ldots, \sigma_{q}\right), \mathbf{O}\right]$, or its transposition, decided by $m>n$ or $m<n$, $\mathbf{O}$ is the zero matrix, and $\sigma_{1} \geq \sigma_{2} \geq \cdots \geq \sigma_{q}, q=\min (m, n)$. $\sigma_{i}(i=$ $1,2, \ldots, q)$ are called the singular values of matrix $\mathbf{A}$.

For a discrete signal $\mathbf{X}=[x(1), x(2), \ldots, x(N)]$, the following matrix can be created:

A

$$
=\left[\begin{array}{cccc}
x(1) & x(2) & \cdots & x(n) \\
x(2) & x(3) & \cdots & x(n+1) \\
\vdots & \vdots & \vdots & \vdots \\
x(N-n+1) & x(N-n+2) & \cdots & x(N)
\end{array}\right]_{m \times n},
$$

where $1<n<N, m=N-n+1$. Then, $\mathbf{A} \in \mathbf{R}^{m \times n}$, and this matrix is called the Hankel matrix of A. Perform SVD on the Hankel matrix. According to (2), matrix A can be transformed into the form of column vectors $\mathbf{u}_{i}$ and $\mathbf{v}_{i}$ :

$$
\mathbf{A}=\mathbf{u}_{1} \sigma_{1} \mathbf{v}_{1}^{T}+\mathbf{u}_{2} \sigma_{2} \mathbf{v}_{2}^{T}+\cdots+\mathbf{u}_{q} \sigma_{q} \mathbf{v}_{q}^{T}
$$

where $\mathbf{u}_{i} \in \mathbf{R}^{m \times 1}$ and $\mathbf{v}_{i} \in \mathbf{R}^{n \times 1}, i=1,2, \ldots, q, q=\min (m, n)$.

Let $\mathbf{A}_{i}=\mathbf{u}_{i} \sigma_{i} \mathbf{v}_{i}^{T}$; then $\mathbf{A}_{i} \in \mathbf{R}^{m \times n}$. Supposing that $\mathbf{P}_{i, 1}$ is the first row vector of $\mathbf{A}_{i}$ and $\mathbf{H}_{i,(n)}$ is the last column of $\mathbf{A}_{i}$ 
except for the first number, $\mathbf{P}_{i, 1} \in \mathbf{R}^{1 \times n}$ and $\mathbf{H}_{i,(n)} \in \mathbf{R}^{(m-1) \times 1}$, as shown below:

$\mathbf{A}_{i}$

$$
=\left[\begin{array}{cccc|}
\hline x_{i}(1) & x_{i}(2) & \cdots & x_{i}(n) \\
\hline x_{i}(2) & x_{i}(3) & \cdots & x_{i}(n+1) \\
\vdots & \vdots & \vdots & \vdots \\
x_{i}(N-n+1) & x_{i}(N-n+2) & \cdots & x_{i}(N)
\end{array}\right]_{m \times n} .
$$

A component signal $\mathbf{P}_{i}$ can then be obtained. The vector form is shown in

$$
\mathbf{P}_{i}=\left(\mathbf{P}_{i, 1}, \mathbf{H}_{i,(n)}^{T}\right) .
$$

For each $\mathbf{A}_{i}$, the component signal $\mathbf{P}_{i}$ can be constructed. All the component signals make up one type of decomposition for the original signal $\mathbf{X}$ and $i$ th $\mathbf{P}_{i}$ corresponds to the $i$ th singular value $\sigma_{i}$.

It can be proved that the original signal $\mathbf{X}$ is equal to the linear superposition of all component signals $\mathbf{P}_{i}$ :

$$
\mathbf{X}=\mathbf{P}_{1}+\mathbf{P}_{2}+\cdots+\mathbf{P}_{q} .
$$

Therefore, the signal can be reconstructed by appropriate singular values to achieve noise reduction and component signal separation.

2.3. Size of the Hankel Matrix. The size of the Hankel matrix plays a key role in signal processing based on SVD. When the length of the original signal is $N$, the column number of the matrix can be any integer in [2,N-1]. To determine $n$ in (2), there is no optimal method yet. Kanjilal et al. proposed the approach using the singular value ratio (SVR) spectrum $[25,26]$. The SVR is defined as $\sigma_{1} / \sigma_{2}$, and the variation of this ratio with the row length $n$ can be used to detect periodicity in a signal. If there are peaks on the SVR spectrum, a periodic component should exist in the signal. Corresponding $n$ can be decided. While there are multiple periodicity components or strong noise in the signal, this method will lose its effect. Zhao and Ye put forward an architecture rule of the Hankel matrix in the literature [23]. If signal length $N$ is even, the column of the matrix $n=N / 2$ and the row $m=N / 2+1$. If $N$ is odd, $n=m=N / 2+1$. Combined with the difference spectrum (interpreted in Section 2.4), the maximum peak can be found. The signal should be reconstructed by the singular values before the peak. This architecture method can achieve the maximum noise removing quantity. However, if the signal contains complex components, some useful components would be filtered. In more general cases, the most effective method is to select different $m$ and $n$ values for trial and error. The characteristic of each component $\mathbf{P}_{i}$ can be observed and analyzed. Referring to some studies about rolling bearing fault feature extraction based on SVD $[15,21$, $22,27]$, the row $m$ is always set to a large number to ensure the frequency spectrum resolution of the component signal. The column $n$ is an integer within 100. To further evaluate the performance of Hankel matrix's size, the following analysis is performed.
For a signal containing noise $x(i)$, it can be written as

$$
x(i)=s(i)+n(i),
$$

where $s(i)$ is the signal and $n(i)$ is the noise. The Hankel matrix of the original signal can be created as follows:

$$
\mathbf{A}=\mathbf{A}_{s}+\mathbf{A}_{n}
$$

where $\mathbf{A}_{s}$ corresponds to $s(i), \mathbf{A}_{n}$ corresponds to $n(i)$, and $\mathbf{A}_{s}, \mathbf{A}_{n} \in \mathbf{R}^{m \times n}$. The size of $\mathbf{A}$ is optimal.

Based on the characteristics of the Hankel matrix, the singular values of $\mathbf{A}_{s}$ can be expressed as $\sigma\left(\mathbf{A}_{s}\right)=$ $\left(\sigma_{s 1}, \sigma_{s 2}, \ldots, \sigma_{s k}, 0, \ldots, 0\right)$, with length $q=\min (m, n)$ and $q>k$. However, for $\mathbf{A}_{n}$ of the noise, its singular values are the form of $\sigma\left(\mathbf{A}_{n}\right)=\left(\sigma_{n}, \sigma_{n}, \ldots, \sigma_{n}\right)$, with length $q=\min (m, n)$. All the singular values are equal to a number.

Supposing that two matrixes A and B exist, (9) can be proven in the literature [24]:

$$
\sigma(\mathbf{A}+\mathbf{B}) \leq \sigma(\mathbf{A})+\sigma(\mathbf{B}) .
$$

Then, (10) can be obtained:

$$
\sigma(\mathbf{A}) \leq\left(\sigma_{s 1}+\sigma_{n}, \ldots, \sigma_{s k}+\sigma_{n}, \sigma_{n}, \ldots, \sigma_{n}\right) .
$$

This result reveals that, for the Hankel matrix $\mathbf{A}$, its back singular values are equal to or smaller than a constant. Therefore, according to the analysis above, if the Hankel matrix size is applicable, a series of singular values that are of the form of (10) can be acquired. The change between the back singular values tends to zero, and there are no obvious peaks in the back part of the difference spectrum. Then, the back singular values represent the noise components. All useful component signals can be reconstructed by the front singular values.

2.4. Study of Difference Spectrum of Singular Value. In (10), the length $q=\min (m, n)$ and it is clear that the last $q-k$ singular values are smaller than the front $k$ ones. There is a sudden change at the $k$ th singular value. Thus, the difference spectrum of singular value is defined as follows:

$$
b_{i}=\sigma_{i}-\sigma_{i+1} \quad(i=1,2, \ldots, q-1) .
$$

The sequence reflects the change between adjacent singular values. When a sudden change occurs at the $k$ th singular value, a peak will appear on the difference spectrum. Find the maximum peak and select the front singular value to reconstruct the signal. Then the noise can be reduced.

Now, another case exists. When the original signal contains different components, the performance on difference spectrum is unknown. For a signal containing two components, it can be written as

$$
x(i)=s_{1}(i)+s_{2}(i),
$$

where $x(i)$ is the signal and $s_{1}(i)$ and $s_{2}(i)$ are the two components. The Hankel matrix can be expressed as

$$
\mathbf{A}=\mathbf{A}_{1}+\mathbf{A}_{2},
$$




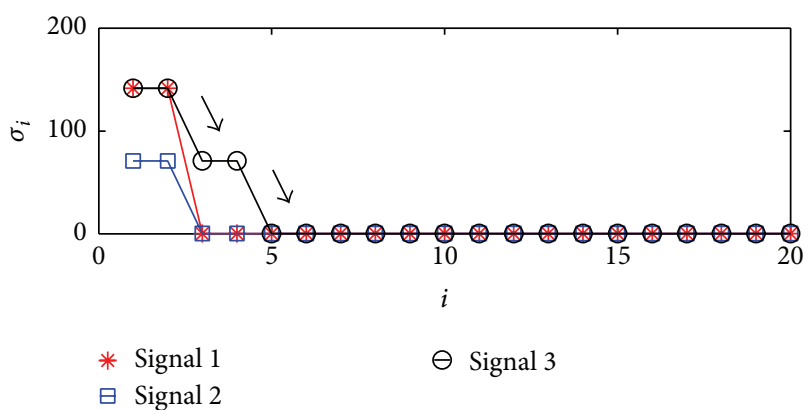

(a)

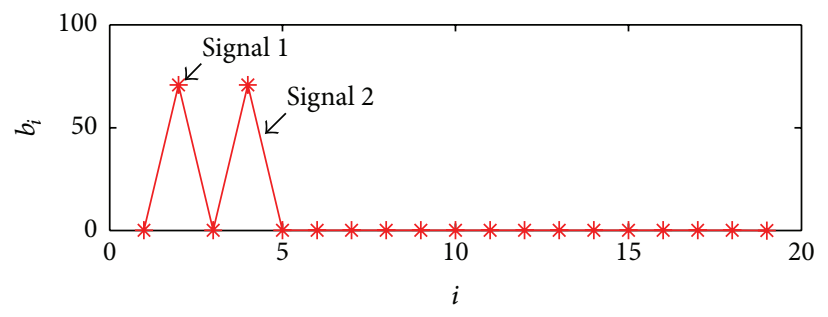

(b)

Figure 1: (a) Curve of singular values and (b) difference spectrum of signal 3.

where $\mathbf{A}_{1}$ corresponds to $s_{1}(i), \mathbf{A}_{2}$ corresponds to $s_{2}(i)$, and $\mathbf{A}, \mathbf{A}_{1}, \mathbf{A}_{2} \in \mathbf{R}^{m \times n}$. The formulas below are established, respectively; consider

$$
\begin{aligned}
& \mathbf{A}_{1}=\mathbf{U}_{1} \Sigma_{1} \mathbf{V}_{1}, \\
& \mathbf{A}_{2}=\mathbf{U}_{2} \Sigma_{2} \mathbf{V}_{2},
\end{aligned}
$$

where $\mathbf{U}_{1}, \mathbf{U}_{2} \in \mathbf{R}^{m \times m}$ and $\mathbf{V}_{1}, \mathbf{V}_{2} \in \mathbf{R}^{n \times n}$. The matrixes $\mathbf{U}_{1}$, $\mathbf{U}_{2}, \mathbf{V}_{1}$, and $\mathbf{V}_{2}$ are orthogonal.

To simplify the situation, assuming $\sigma\left(\mathbf{A}_{1}\right)=\left(\sigma_{11}, \sigma_{12}\right.$, $0, \ldots, 0), \sigma\left(\mathbf{A}_{2}\right)=\left(\sigma_{21}, \sigma_{22}, 0, \ldots, 0\right)$ and $\sigma_{11} \geq \sigma_{12} \geq \sigma_{21} \geq$ $\sigma_{22}$, matrix $\mathbf{A}$ can be transformed into

$$
\mathbf{A}=\mathbf{u}_{11} \sigma_{11} \mathbf{v}_{11}^{T}+\mathbf{u}_{12} \sigma_{12} \mathbf{v}_{12}^{T}+\mathbf{u}_{21} \sigma_{21} \mathbf{v}_{21}^{T}+\mathbf{u}_{22} \sigma_{22} \mathbf{v}_{22}^{T} .
$$

If the two component signals are uncorrelated, $\mathbf{U}_{1}$ and $\mathbf{U}_{2}$ are still orthogonal. The situation for $\mathbf{V}_{1}$ and $\mathbf{V}_{2}$ is similar. Hence, $\sigma(\mathbf{A})=\left(\sigma_{11}, \sigma_{12}, \sigma_{21}, \sigma_{22}, 0, \ldots, 0\right)$.

For example, there are signal $1 f_{1}(t)=2 \sin (2 \pi * 200 t)$, signal $2 f_{2}(t)=\sin (2 \pi * 100 t)$, and signal $3 f_{3}(t)=$ $f_{1}(t)+f_{2}(t)$. Signal 1 and signal 2 are the components of signal 3. Three Hankel matrixes can be established for $f_{1}(t), f_{2}(t)$, and $f_{3}(t)$. The singular value sequences of the three signals are illustrated in Figure 1(a). In this figure, it is clear that the singular values $\sigma_{1}, \sigma_{2}$ of signal 3 are equal to that of signal 1 . The singular values $\sigma_{3}, \sigma_{4}$ of signal 3 are equal to that of signal 2 . Therefore, when an original signal contains different components that have poor correlation, its singular value sequences can reflect the singular values of component signals. In this case, the component signal 1 can be reconstructed by $\sigma_{1}, \sigma_{2}$ of signal 3. The component signal 2 can be rebuilt by $\sigma_{3}, \sigma_{4}$ of signal 3 . Because the energy of the component signals is different, there is a sudden change between $\sigma_{2}$ and $\sigma_{3}$. Corresponding to the difference spectrum of signal 3 shown in Figure 1(b), there are two peaks. One appears in the second coordinates. The other is located in the fourth singular value. Thus, the number of main components of the signal can be observed according to the peak on the difference spectrum. Then, every component can be reconstructed by the singular value at the peak point and the point of the front valley.

A simulation analysis will be discussed subsequently. When a bearing fault occurs, the components of the vibration
TABLE 1: Parameters of fault simulated signal.

\begin{tabular}{lcccccc}
\hline$\alpha$ & $T$ & $f_{m} / \mathrm{Hz}$ & $f_{s} / \mathrm{Hz}$ & $f_{c 1} / \mathrm{Hz}$ & $f_{c 2} / \mathrm{Hz}$ & $\mathrm{SNR}$ \\
\hline 800 & $1 / 16000$ & 156 & 30 & 7000 & 2000 & -11 \\
\hline
\end{tabular}

signal on casing will be complex. Taking into account the main components, such as the bearing fault signal, the vibration signal caused by the rotor, and the environmental noise, the simulated signal can be expressed as

$$
\begin{aligned}
x(t)= & A e^{-\alpha t} * \sin 2 \pi f_{c 1} n T+B \sin 2 \pi f_{s} n T \\
& * \sin 2 \pi f_{c 2} n T+C \zeta(n), \\
t= & \bmod \left(n T, \frac{1}{f_{m}}\right),
\end{aligned}
$$

where $\alpha$ is the exponent factor and $T$ is the sampling interval. $n$ is the sampling points. $f_{m}$ is related to the fault characteristic frequency of the bearing. $f_{s}$ is the rotation frequency of the rotor. $f_{c 1}$ and $f_{c 2}$ are the carrier frequencies, which reflect the resonance of the system. $\zeta(n)$ is the white noise chosen from the Gauss distribution. All the parameters of the simulation signal are listed in Table 1.

The simulated fault signal on the casing with an SNR of $-11, A=2$, and $B=1$ is shown in Figure 2(a). The time waveform shows weak fault impulse features and frequency modulation information. A Hankel matrix with row $m=$ 20000 and column $n=40$ is created. After this matrix is processed by SVD, 40 singular values are acquired corresponding to 40 component signals. The difference spectrum of singular value is illustrated in Figure 2(b). According to the preceding study, it can be inferred that there are two peaks representing the vibration signal caused by the rotor and bearing fault signal on the difference spectrum. Four component signals rebuilt by the front 4 singular values are shown in Figure 3. Add the front two component signals. As shown in Figure 4(a), it is clear that the value of $60.68 \mathrm{~Hz}$ is related to the rotational frequency of the rotor on the envelope spectrum. Then, $P_{3}$ and $P_{4}$ are added together and the envelope spectrum is shown in Figure $4(\mathrm{~b})$. The fault feature frequency of the bearing, $156.5 \mathrm{~Hz}$ and $313 \mathrm{~Hz}$, can be easily extracted. 


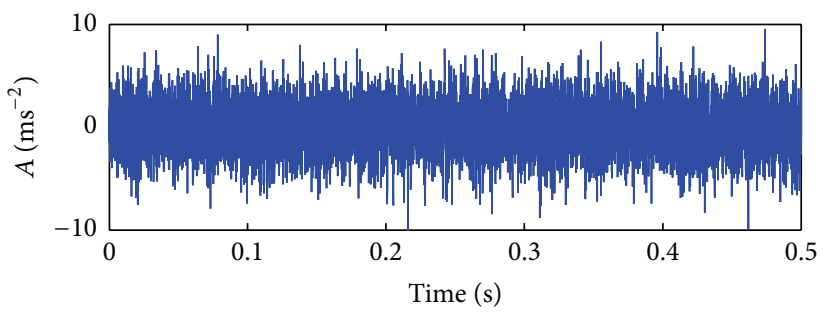

(a)

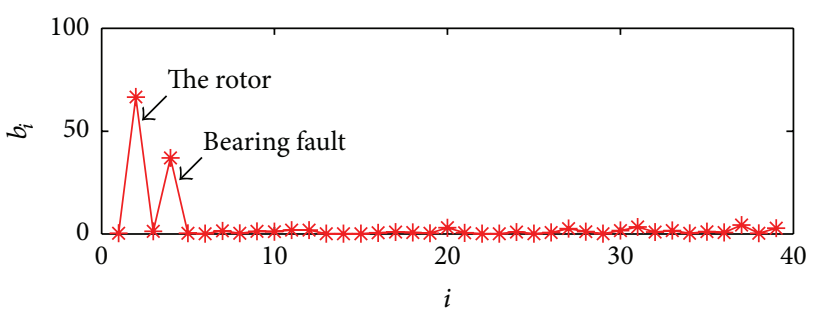

(b)

FIgURE 2: (a) Simulated fault signal and (b) difference spectrum of singular value.

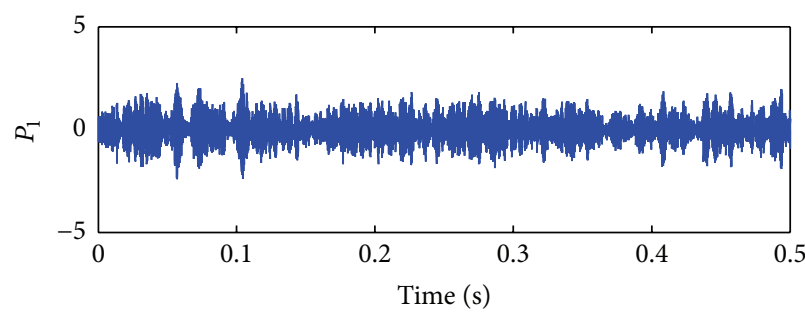

(a)

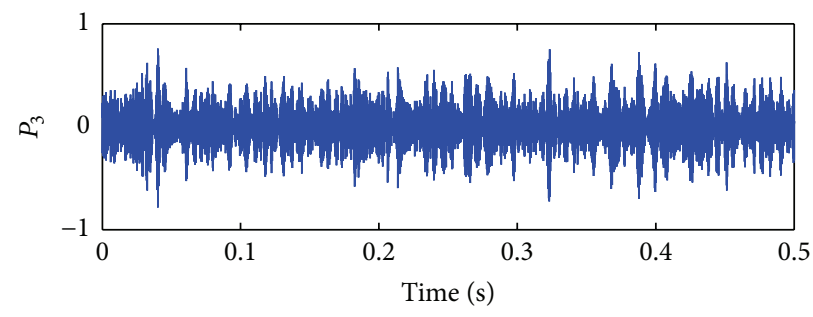

(c)

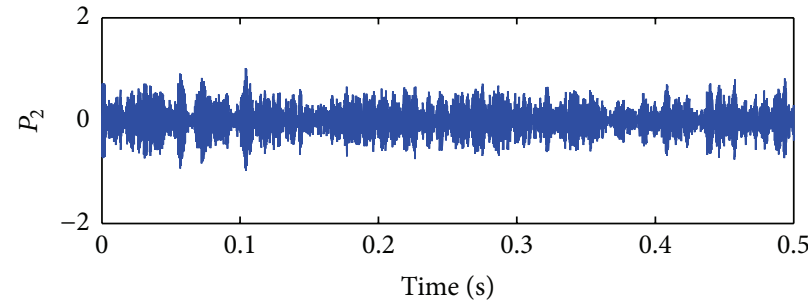

(b)

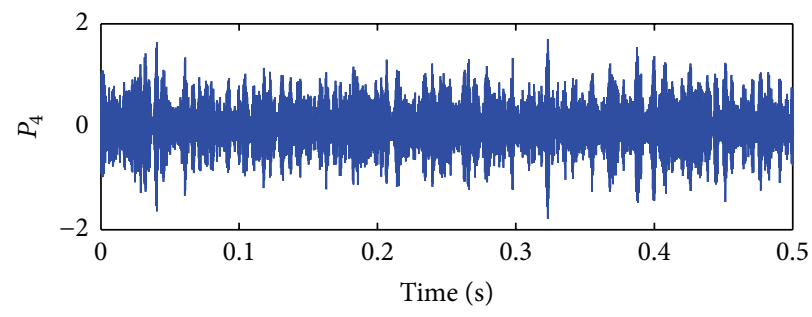

(d)

Figure 3: Component signals $P_{1}, P_{2}, P_{3}$, and $P_{4}$ of the simulated fault signal by SVD.

2.5. Correlated Kurtosis. The kurtosis index can effectively reflect the impact characteristic of the vibration signals. Hence, many researchers estimate the impact level by means of kurtosis in rolling bearing fault diagnosis. However, there are many other impact sources in rotating machinery such as gear pitting, rub-impact faults, and shaft cracking. Therefore, the kurtosis will fail to measure the rolling bearing fault signal sometimes. To improve this weakness, the correlated kurtosis (CK) is put forward [28]. The calculation formula of the CK is as follows:

$$
\mathrm{CK}(T)=\frac{\sum_{n=1}^{N}\left(y_{n} y_{n-T}\right)^{2}}{\left(\sum_{n=1}^{N} y_{n}^{2}\right)^{2}},
$$

where $y_{n}$ is a signal sequence, $N$ is the sample length, and $T$ is the cycle of the pulse signal. When the cycle parameter $T$ is zero, the CK is degraded to kurtosis. The CK contains the characteristics of the kurtosis and correlation function. Compared to the kurtosis, the CK can reflect the impact level of a vibration signal with a certain period more accurately. When the CK serves as fault feature value, the fault period $T$ can be set to the rolling bearing fault period. The CK of the components whose impact period is same as the rolling bearing fault period is greater than the other uncorrelated ones. Thus, the CK value can be used to judge the component signals that represent rolling bearing failure.

\section{Fault Feature Extraction Strategy}

3.1. The Method Based on EMD and Difference Spectrum of Singular Value. Based on the analysis above, firstly, a Hankel matrix for a signal can be created. Secondly, the difference spectrum of singular value can be obtained after the matrix is processed by SVD. Finally, the component signals can be reconstructed by the singular value on the peak point and the front valley point. This approach can adaptively decompose the signal to extract components and reduce noise. While bearing failure occurs at an early stage for aeroengines, the fault signal will be weak on the casing, and the energy of the fault signal will be smaller than the other components. Hence, the peak related to the fault signal will be inconspicuous. As shown in Figure 2(b), if the energy of the vibration signal caused by the rotor increases, the height of the first peak will be more obvious. It is not easy to confirm the presence of the second peak, which represents the bearing fault information.

To solve this problem, assuming the fault signals are produced by fault impulses and the carrier frequency is the high resonance frequency band of the system, this paper 


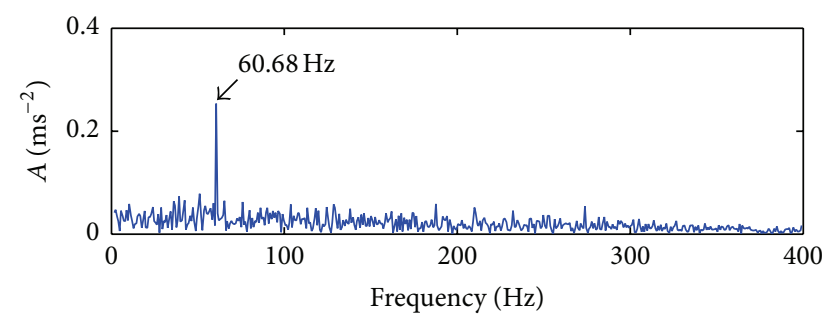

(a)

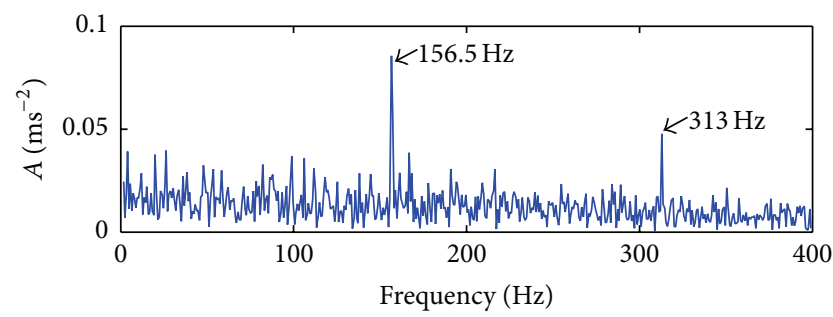

(b)

FIGURE 4: (a) Envelope spectrum of the reconstructed signal by the first two singular values and (b) envelope spectrum of the reconstructed signal by the third and fourth singular values.

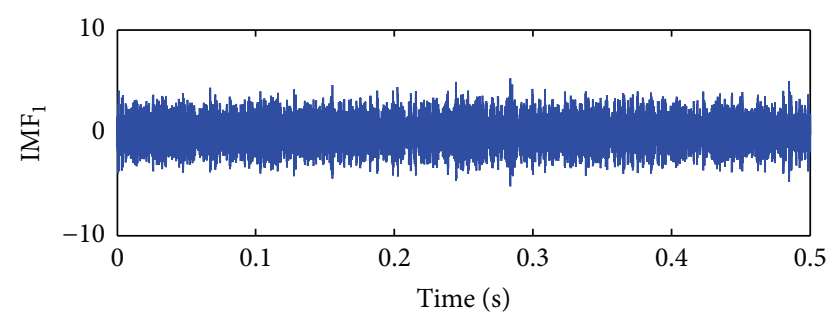

(a)

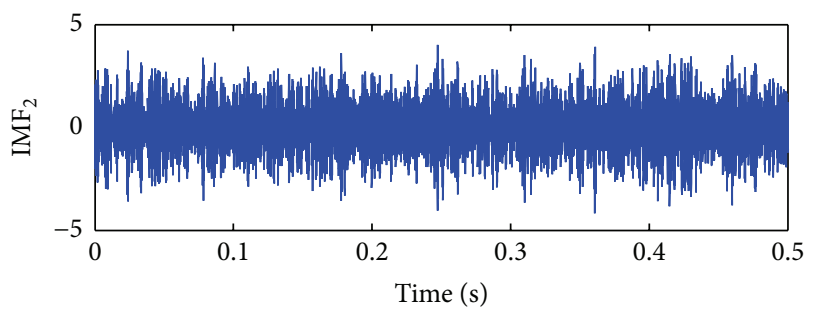

(c)

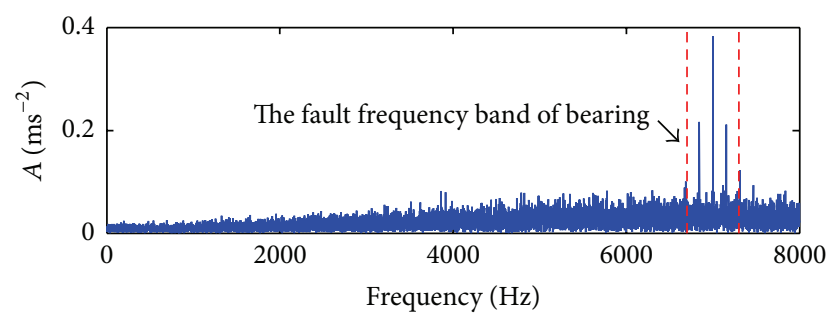

(b)

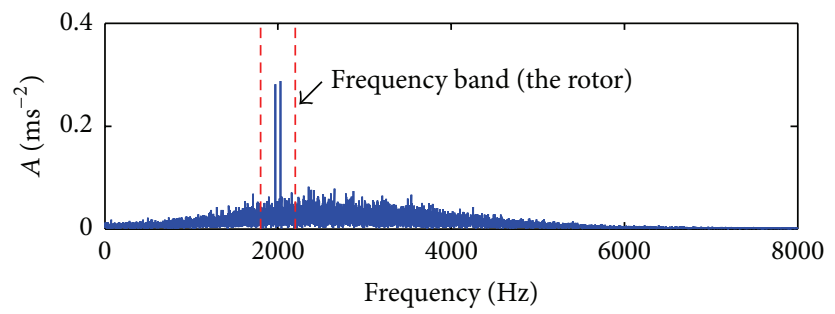

(d)

FIGURE 5: EMD process. (a) The first IMF by EMD. (b) The frequency spectrum of IMF1. (c) The second IMF by EMD. (b) Frequency spectrum of IMF2.

treats EMD as a preprocessing method. A series of IMFs can be obtained after EMD. Select the IMFs representing the high frequency band. The information of bearing fault is always included in them. Then, handle these IMFs with the method based on SVD and the difference spectrum.

The simulated fault signal on casing above is processed by EMD. The time-domain waveforms of IMF1 and IMF2 are illustrated in Figures 5(a) and 5(c). Figures 5(b) and 5(d) show the frequency spectra of IMF1 and IMF2, respectively. One can see that IMF1 contains the fault frequency band of the bearing, and the frequency band of the vibration signal caused by the rotor is decomposed to IMF2. Furthermore, Figure 6 is the envelope spectrum of IMF1. The fault feature frequency of the bearing cannot be extracted because of the strong noise. A Hankel matrix with row $m=20000$ and column $n=50$ is created. 50 singular values are acquired by SVD. The difference spectrum is shown in Figure $7(a)$. We can infer that there is only one peak related to the bearing fault. Figure 7(b) is the signal reconstructed by the front two singular values, and Figure 7(c) shows its envelope spectrum. The fault feature frequencies of the bearing are found to be $156.4 \mathrm{~Hz}$ and $312.8 \mathrm{~Hz}$.

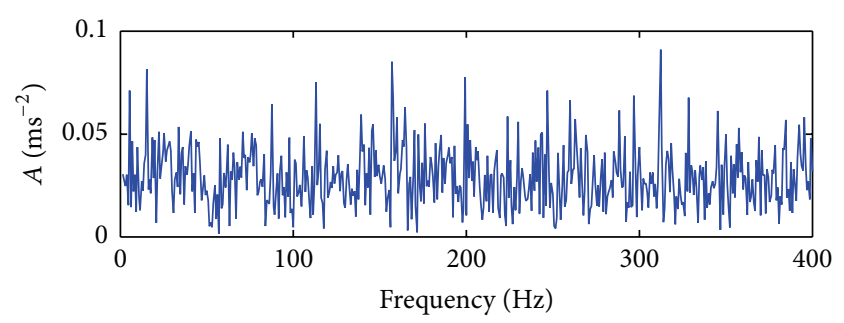

Figure 6: Envelope spectrum of IMF1.

3.2. The Procedures of the Method Based on EMD and Difference Spectrum of Singular Value. In this paper, a method based on EMD and difference spectrum of singular value for the fault diagnosis of rolling bearings is proposed. The specific process is illustrated in Figure 8 . The procedures include the following.

(1) The Procedure of EMD. Process the vibration signal with EMD, obtain the frequency spectrum of every IMF, and select the IMF related to the high frequency band. 


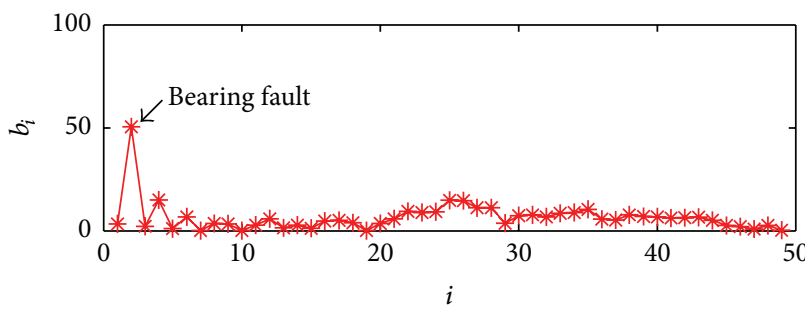

(a)

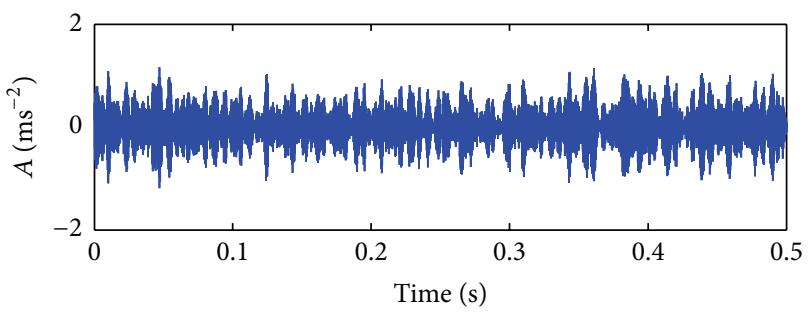

(b)

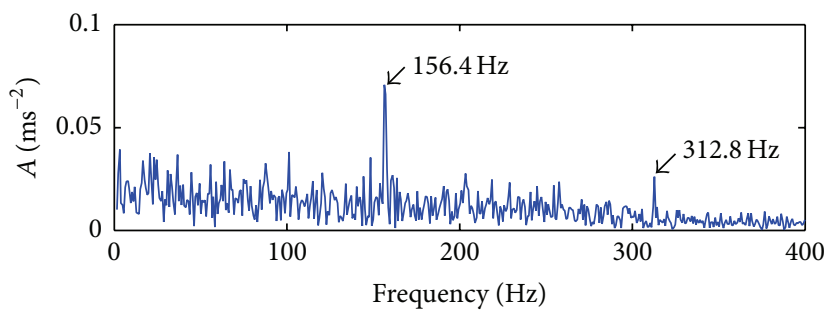

(c)

FIgURE 7: Processing for IMF1. (a) Difference spectrum. (b) Time waveform reconstructed by the first two singular values. (c) Envelope spectrum of the reconstructed signal.

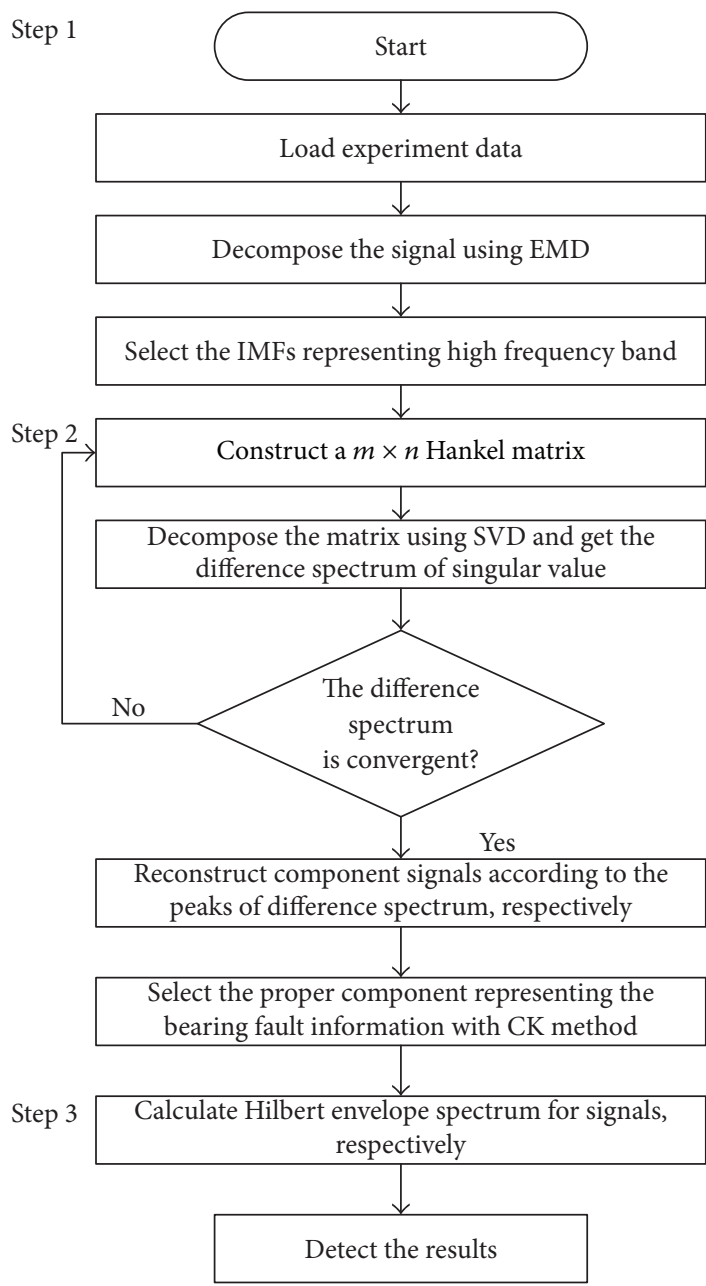

FIGURE 8: Signal processing procedures.
(2) The Procedure of SVD. (a) Create a Hankel matrix with row $m$ and column $n$ for selected IMF signal. (b) Process the matrix by SVD, and calculate the difference spectrum of singular value. (c) Observe the convergence of the difference spectrum at the end. If there are obvious peaks on the back part of the difference spectrum, change $n$ and repeat the procedure of creating a Hankel matrix and SVD. (d) Choose the singular value at the peak point and the front valley point to reconstruct a component signal according to the peak on the difference spectrum. (e) If there are several peaks on the spectrum, the CK index can be utilized for the selection criterion from these components.

(3) Envelope Demodulation. Use the envelope demodulation method to process the component signal. Detect the fault feature frequency of the rolling bearing and judge the state of the bearing.

\section{Engineering Applications}

4.1. The Fault Simulation Experiment of Rolling Bearing and the Signal Process. The rolling bearing fault simulation test rig based on casing can be observed in Figure 9. The rotor is connected to a motor by a coupling. A fan is located on the other side of the rotor. The rolling bearing and bearing block support the rotor. The relationship between the bearing block and outer race of the bearing is an interference fit. Similar relationship exists between the inner race and the rotor. The bearing block is fixed on the casing with braces and bolt connections.

Considering the situation that sensors are placed on aeroengine casing in practice, accelerometers are installed on casing, except the bearing block in this experiment. The data acquisition system mainly includes accelerometers, filter 

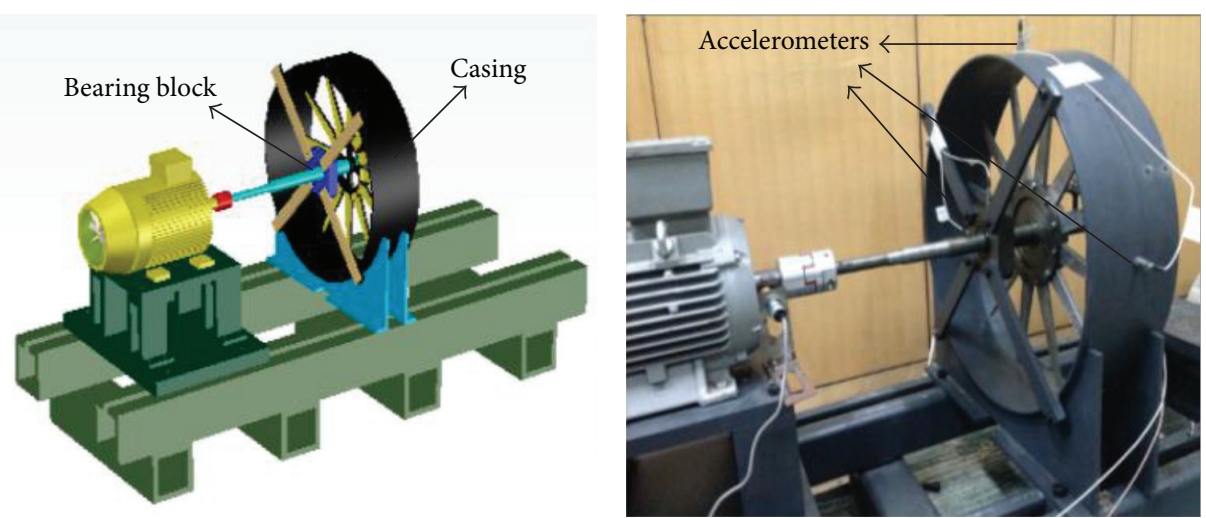

FIGURE 9: The structure of the test rig.

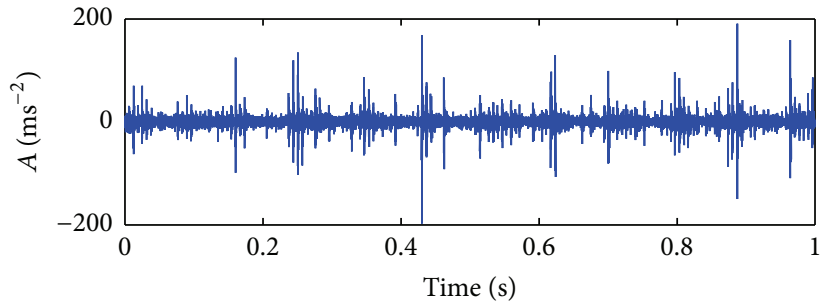

(a)

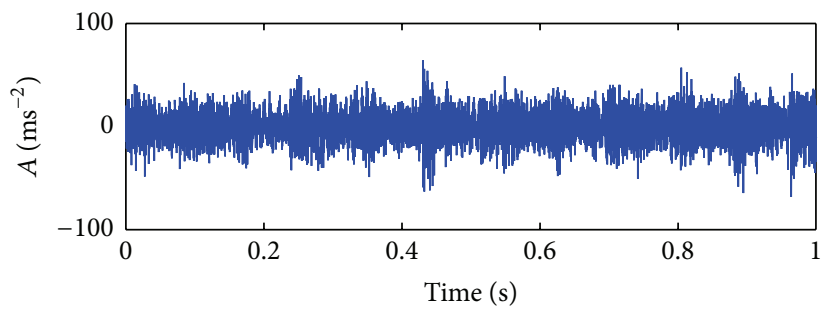

(c)

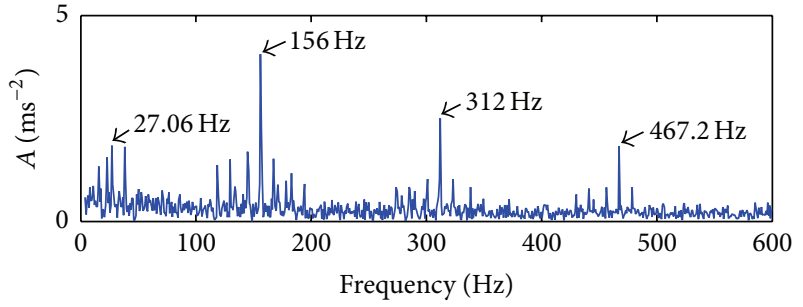

(b)

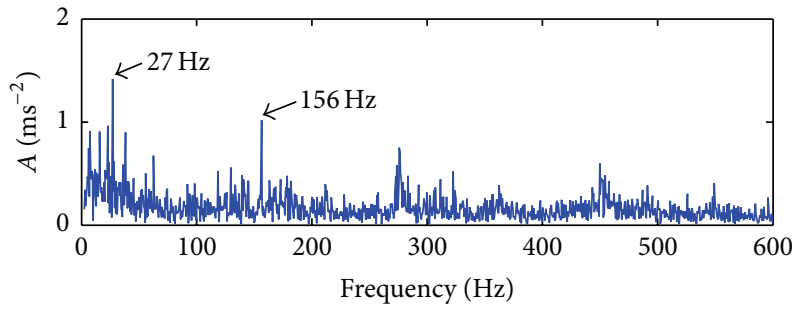

(d)

FIGURE 10: Fault signal of crack width of $1.5 \mathrm{~mm}$. (a) Time waveform of the bearing block. (b) Envelope spectrum of the bearing block. (c) Time waveform of the casing. (d) Envelope spectrum of the casing.

instruments, and an MPS-140801M data acquisition card. Adjust the motor speed to experiment speed 1600 RPM with the inverter, and the sampling rate is $16 \mathrm{KHz}$. The signal length is 20480 points to ensure the resolution of the frequency spectrum.

The test bearings are N206EM, cylindrical roller bearings. The specific parameters of bearings are as follows: inside diameter: $30 \mathrm{~mm}$, outside diameter: $62 \mathrm{~mm}$, pitch diameter: $46 \mathrm{~mm}$, roller diameter: $7.6 \mathrm{~mm}$, the number of rolling elements: 14, and contact angle: 0 degrees. The crack fault is produced in the outer race of the bearings using wire cutting technology. This paper simulated 3 types of outer race failure states, which are slight fault, fault, and serious fault. The crack depth of the 3 conditions is $0.5 \mathrm{~mm}$, and the widths are $0.8 \mathrm{~mm}, 1 \mathrm{~mm}$, and $1.5 \mathrm{~mm}$, respectively. The fault characteristic frequency of the outer race can be calculated by

$$
f_{o}=\frac{1}{2} Z\left(1-\frac{d}{D} \cos \alpha\right) f_{s}
$$

where $f_{s}$ is the rotational frequency, $d$ and $D$ are the roller diameter and pitch diameter, respectively, $\alpha$ is the contact angle, and $Z$ is the number of rolling elements. The result is $f_{o}=155.8 \mathrm{~Hz}$.

To validate whether the conventional envelope demodulation method, which is widely used in engineering, is effective for the vibration signal measured on the casing, a comparison and analysis are conducted. Figures 10-12 are, respectively, the time-domain waveforms and envelope spectra for the vibration signals of the bearing block and casing in the 3 fault conditions.

It is obvious to find the fault characteristic frequencies $1 X, 2 X$, and $3 X$ on the envelope spectra for the vibration signals of the bearing block in the three figures. However, for the vibration signals of the casing, the fault feature can be extracted only in the condition of a serious fault with crack width of $1.5 \mathrm{~mm}$. In the other two states, it is difficult to extract any effective fault features. Only the rotational frequency is obvious. Thus, the main components of vibration signal on 


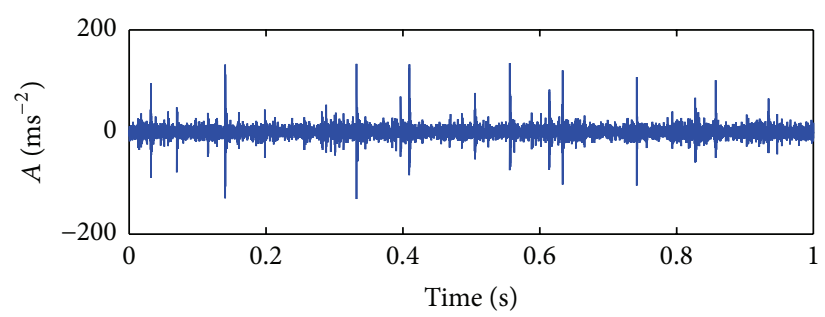

(a)

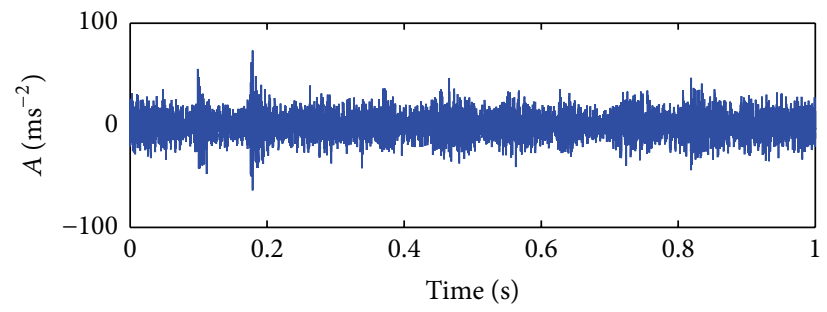

(c)

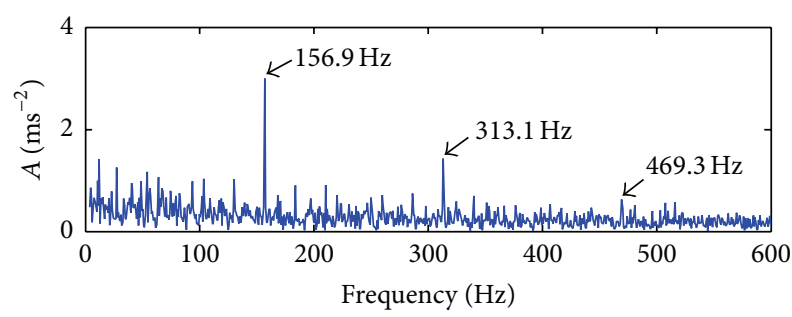

(b)

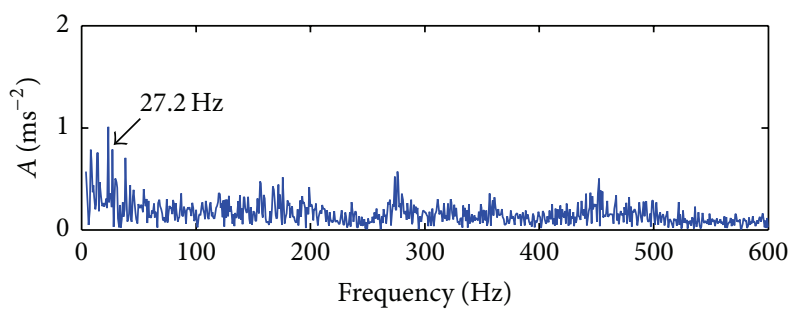

(d)

FIGURE 11: Fault signal of crack width of $1 \mathrm{~mm}$. (a) Time waveform of the bearing block. (b) Envelope spectrum of the bearing block. (c) Time waveform of the casing. (d) Envelope spectrum of the casing.

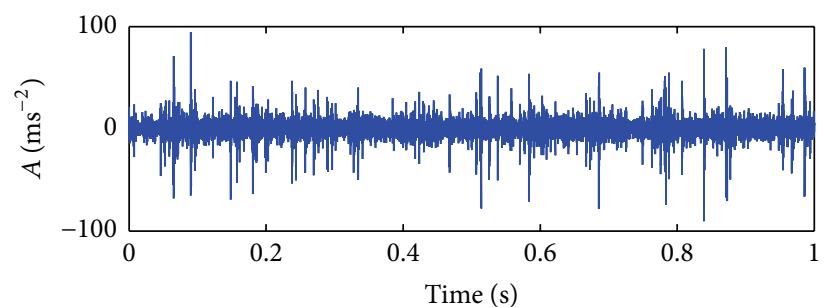

(a)

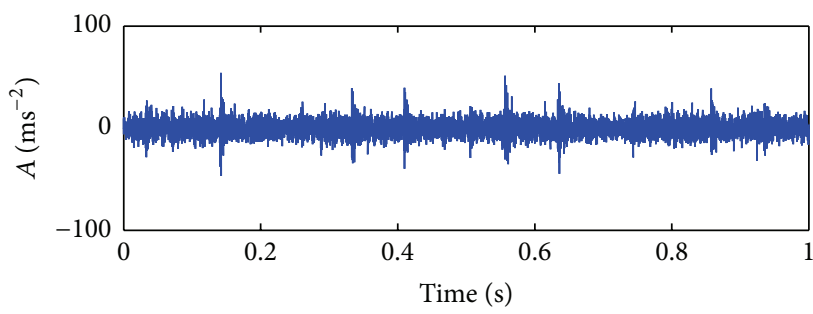

(c)

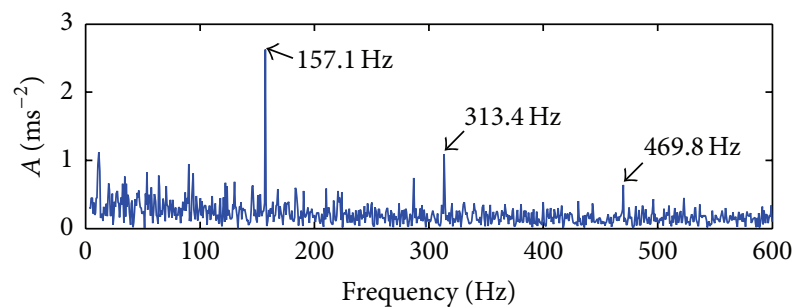

(b)

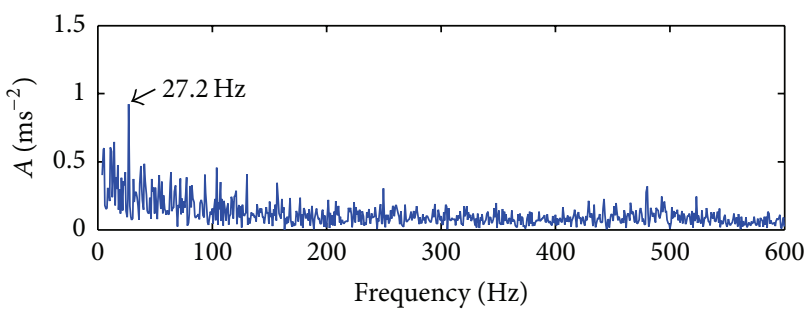

(d)

FIGURE 12: Fault signal of crack width of $0.8 \mathrm{~mm}$. (a) Time waveform of the bearing block. (b) Envelope spectrum of the bearing block. (c) Time waveform of the casing. (d) Envelope spectrum of the casing.

the casing are the modulation signals caused by rotor. The fault signal of the bearing is completely submerged in the other components and noise when the fault of the bearing is slight.

4.2. Fault Feature Extraction of the Vibration Signals Based on Casing. To verify the proposed method in this paper, the vibration signal of the casing on the condition of crack widths of $1 \mathrm{~mm}$ and $0.8 \mathrm{~mm}$ will be discussed. Figures 13(a)-13(c), and $14(\mathrm{a})-14(\mathrm{c})$ are the first three IMF time waveforms for the crack widths of $1 \mathrm{~mm}$ and $0.8 \mathrm{~mm}$, respectively, by EMD. Figures 13(d)-13(f) and 14(d)-14(f) show their frequency spectra. From this step, one can see that the high frequency information (over $4000 \mathrm{~Hz}$ ) of the signal is included in IMF1. Therefore, select IMF1 of the two states to create a Hankel matrix. Choose row $m=20000$ to ensure the resolution of the frequency spectrum. The difference spectrum will be convergent when $\mathrm{n}$ is greater than 60 (crack width of $1 \mathrm{~mm}$ ) and 70 (crack width of $0.8 \mathrm{~mm}$ ). The difference spectra for crack widths of $1 \mathrm{~mm}$ and $0.8 \mathrm{~mm}$ are shown in Figures 15(a) and $15(\mathrm{~b})$, respectively.

There are four obvious peaks on the difference spectra (crack widths of $1 \mathrm{~mm}$ and $0.8 \mathrm{~mm}$ ). Reconstruct the component signals according to the peaks and obtain the 4 main components under the two conditions. Based on the CK method illustrated in (18), $T$ can be set to the rolling bearing 


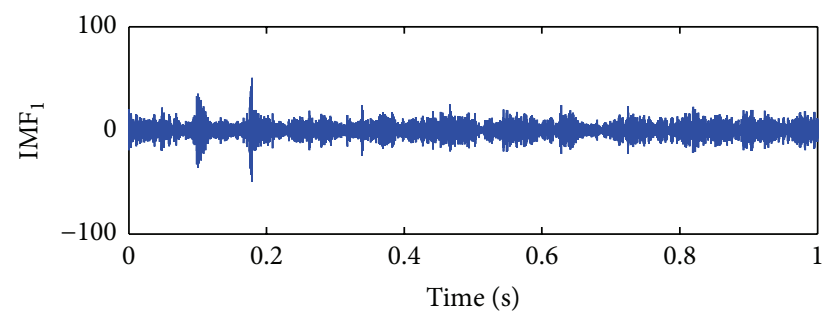

(a)

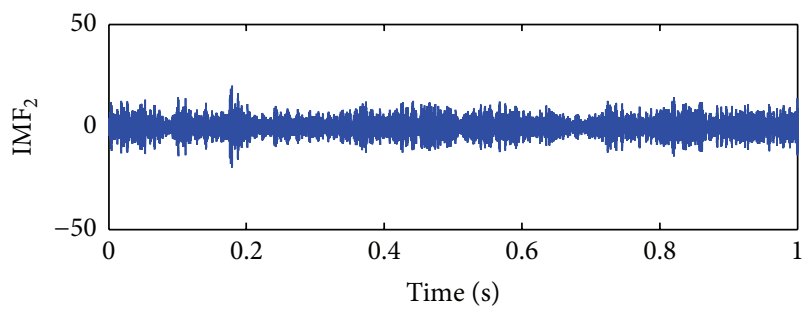

(b)

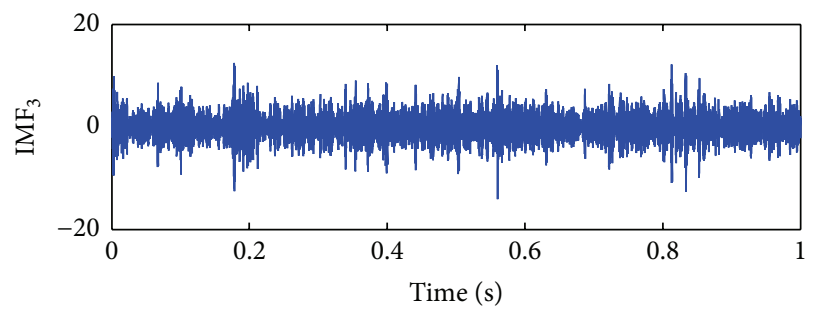

(c)

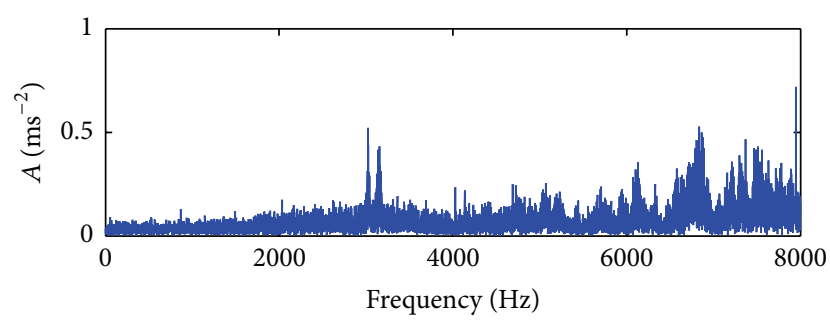

(d)

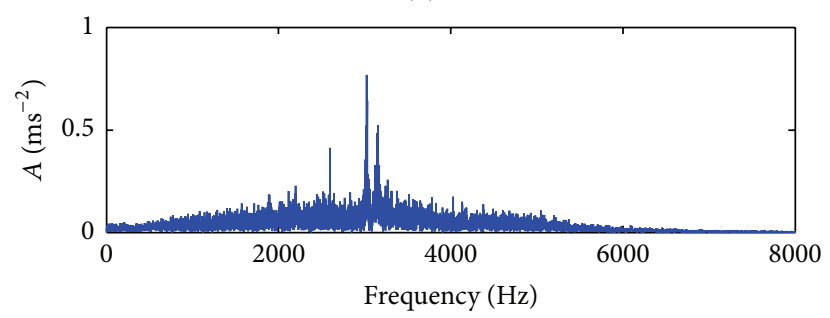

(e)

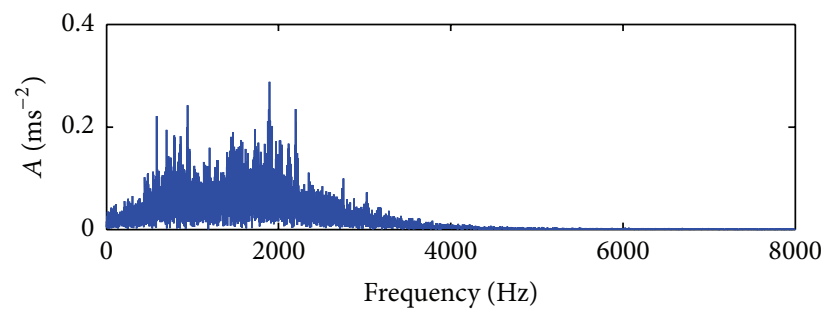

(f)

FIGURE 13: EMD processing of the fault signal for a crack width of $1 \mathrm{~mm}$. (a) IMF1. (b) IMF2. (c) IMF3. (d) Frequency spectrum of IMF1. (e) Frequency spectrum of IMF2. (f) Frequency spectrum of IMF3.

fault period $1 / f_{o}$, and the correlated kurtosis value of each component is shown in Figure 16. It is clear that the CK value of the second component in Figure 16(a) is higher than that of the other ones. In Figure 16(b), the CK value of the fourth component is the largest. We should select the second and fourth components which are related to the bearing fault information for the two cases.

The time waveforms of the reconstructed signal (crack widths of $1 \mathrm{~mm}$ and $0.8 \mathrm{~mm}$ ) are illustrated in Figures 17(a) and 18(a). Figures 17(b) and 18(b) are their frequency spectra, and Figures 17(c) and 18(c) are their envelope spectra. In Figure 17(a), the time waveform has a weak impulse and frequency modulation feature. At the same time, we can observe that the fault frequency band of the bearing is near $7500 \mathrm{~Hz}$ from its frequency spectrum in Figure 17(b). Finally, the fault characteristic frequency $156.3 \mathrm{~Hz}$ can be extracted accurately from envelope spectrum in Figure 17(c). The other component signals and noise are effectively weakened. Likewise, from the detection results for a crack width of $0.8 \mathrm{~mm}$, one can see that the fault frequency band is in the vicinity of $6000 \mathrm{~Hz}$ in Figure 18(b). It is clear to find the fault characteristic frequency $157.1 \mathrm{~Hz}$ on the envelope spectrum in Figure 18(c).

The envelope spectra from the other three peaks of Figure 15 are also illustrated in Figures 19 and 20. Only in Figure 19(a), we can observe the slight fault characteristics. It is difficult to extract the fault frequency from these envelope spectra clearly. However, we can find the obvious rotational frequency and some low frequency components. In other words, the component signals represented by the other three peaks are related to the shaft rotation and other mechanical structure characteristics. Therefore, the components of the vibration signal on the casing are complex. The method proposed in this paper has an excellent ability for adaptive decomposition and fault feature extraction. This method has a practical application value in engineering.

\section{Conclusions}

In this paper, a novel signal processing method based on EMD and difference spectrum of singular value is put forward for the rolling bearing fault diagnosis of vibration signals on casing. Summarizing the study and results mentioned above, the following conclusions can be drawn:

(1) A signal can be adaptively decomposed into a linear sum of component signals using SVD. When the structure of the Hankel matrix is proper, the peaks on the difference spectrum of singular value represent a series of component signals that are uncorrelated. The component signals can be reconstructed according to the peaks, and the effective component related 


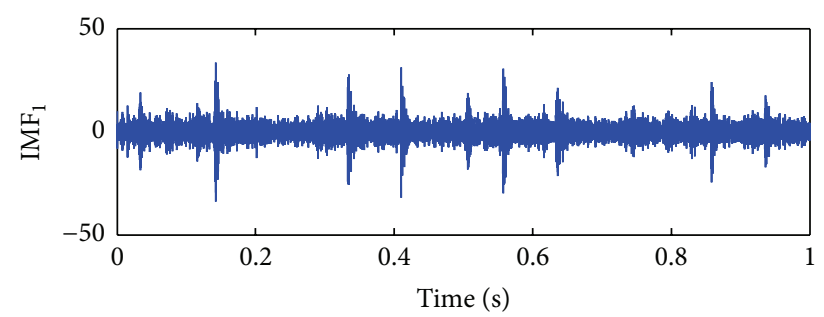

(a)

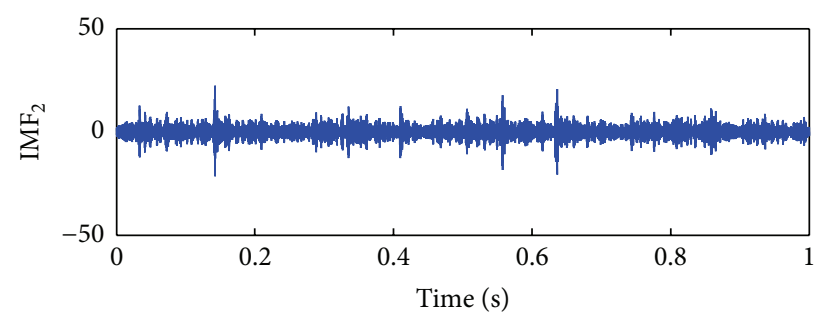

(b)

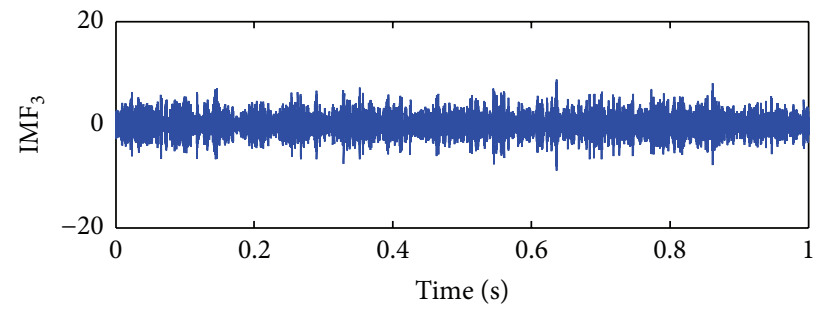

(c)

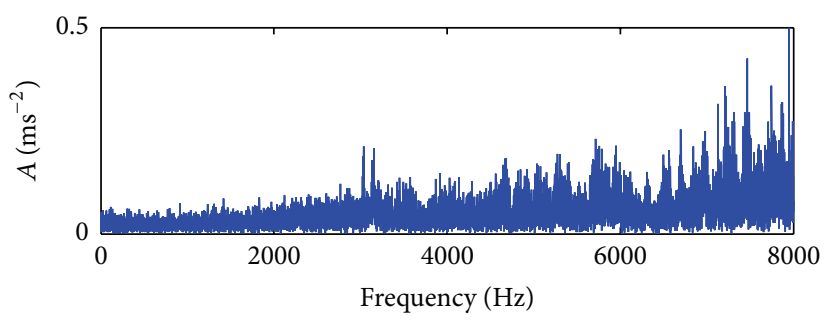

(d)

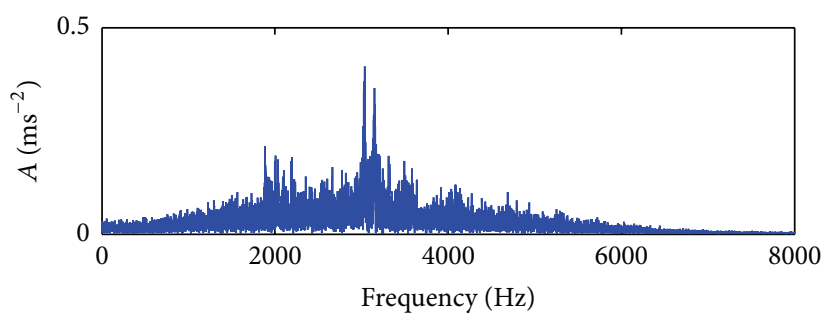

(e)

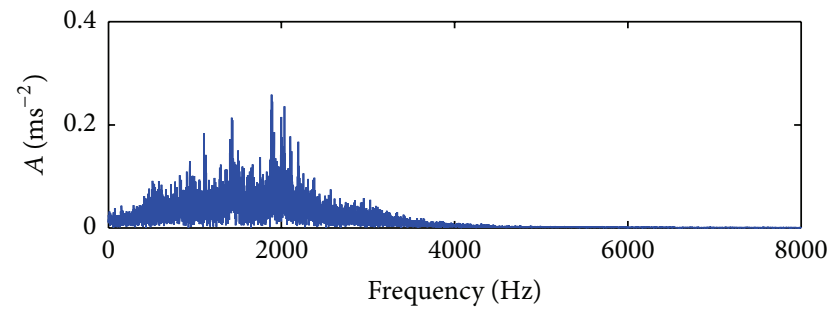

(f)

FIGURE 14: EMD processing of the fault signal for a crack width of $0.8 \mathrm{~mm}$. (a) IMF1. (b) IMF2. (c) IMF3. (d) Frequency spectrum of IMF1. (e) Frequency spectrum of IMF2. (f) Frequency spectrum of IMF3.

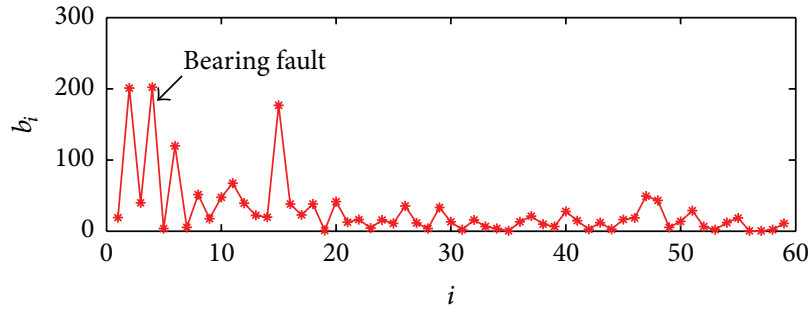

(a)

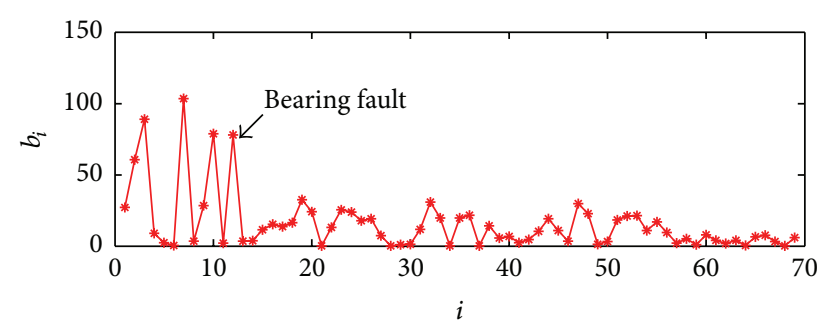

(b)

FIGURE 15: Difference spectrum of singular value. (a) Crack width of $1 \mathrm{~mm}$ and (b) crack width of $0.8 \mathrm{~mm}$.

to rolling bearing fault can be selected by the CK criterion.

(2) Considering the situation in which the components of the vibration signal on the casing are complex, the peaks on the difference spectrum cannot accurately represent the bearing fault when the energy of the fault signal is weak. Therefore, the signal is preprocessed by EMD. The bearing fault information is always included in the IMFs representing the high frequency band. Choose these IMFs, and the effective peak will be more evident on the difference spectrum. The digital simulation result proves the validity of this method.
(3) The fault simulation experiment of rolling bearings is performed for the acquisition of fault vibration signals on the bearing block and casing. When the fault crack is small, the fault feature cannot be extracted by the commonly used envelope demodulation method in engineering. The data analysis results show that the proposed method can exactly separate the fault component signal from the vibration signal on the casing.

\section{Competing Interests}

The authors declare that they have no competing interests. 


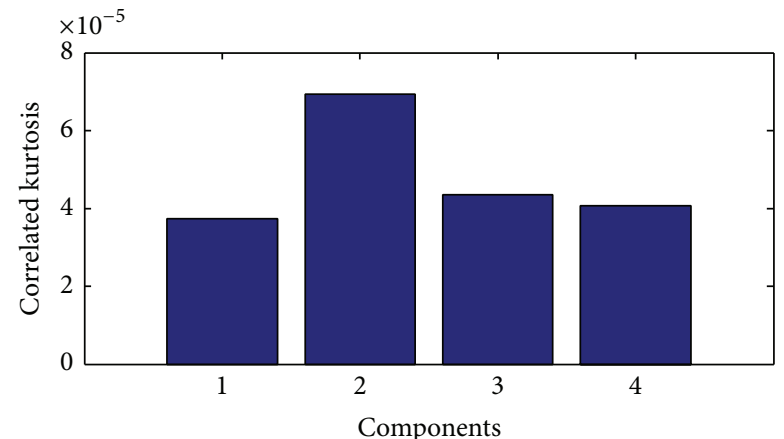

(a)

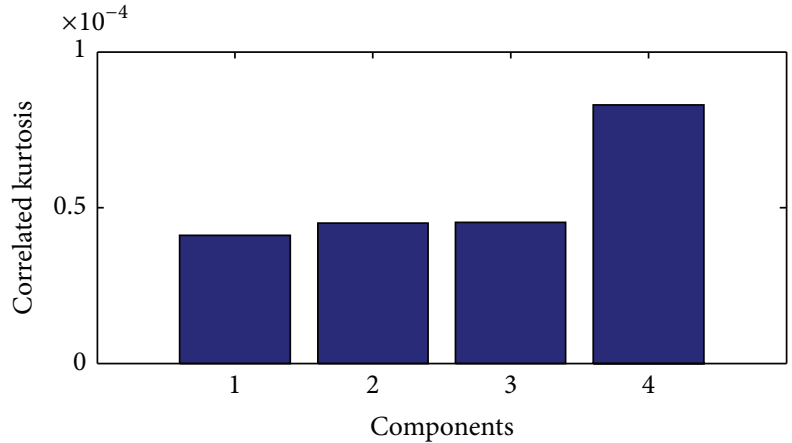

(b)

FIGURE 16: Correlated kurtosis of the 4 component signals. (a) Crack width of $1 \mathrm{~mm}$ and (b) crack width of $0.8 \mathrm{~mm}$.

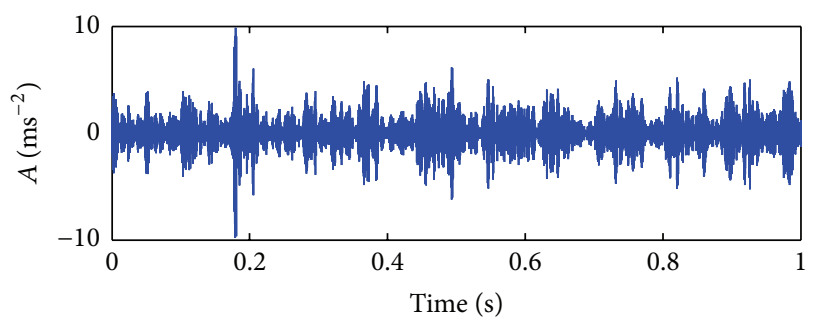

(a)

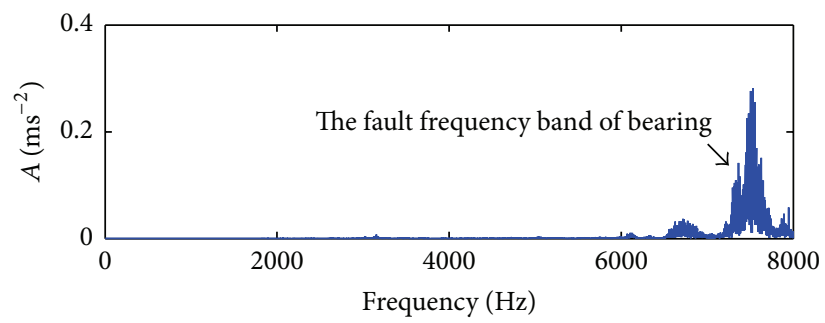

(b)

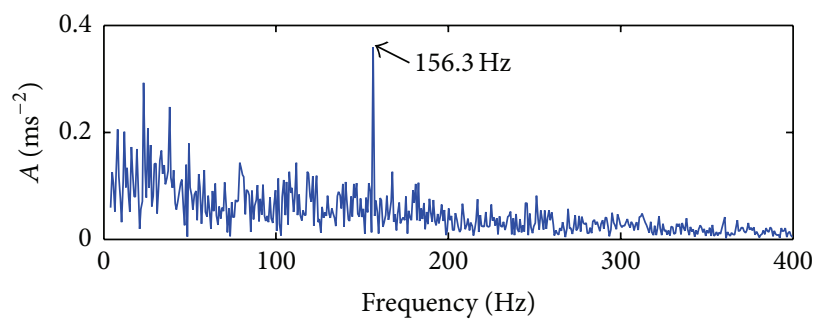

(c)

FIgURE 17: Reconstructed fault signal for a crack width of $1 \mathrm{~mm}$. (a) Time waveform. (b) Frequency spectrum. (c) Envelope spectrum.

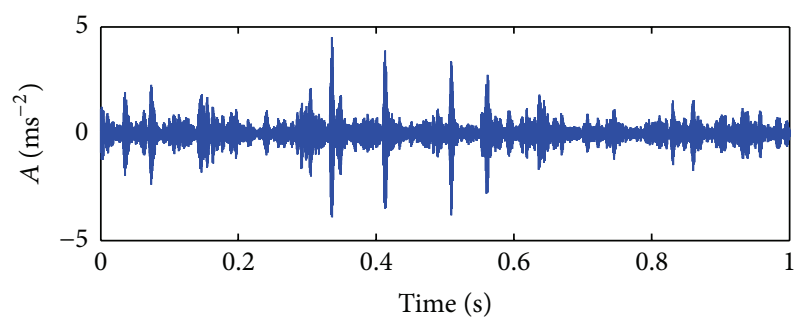

(a)

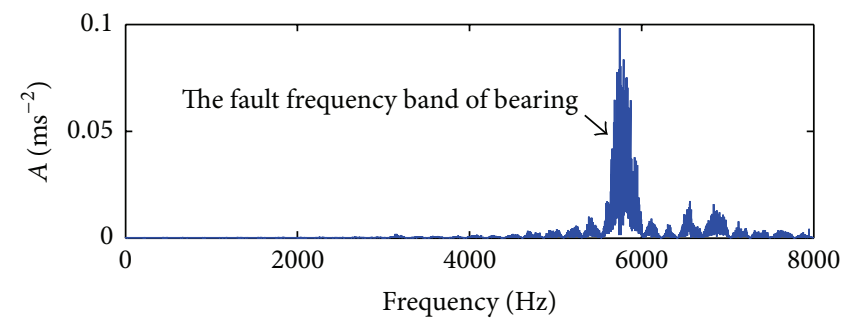

(b)

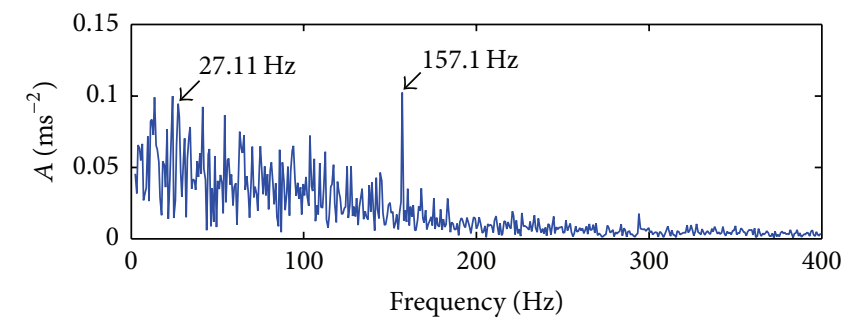

(c)

FIGURE 18: Reconstructed fault signal for a crack width of $0.8 \mathrm{~mm}$. (a) Time waveform. (b) Frequency spectrum. (c) Envelope spectrum. 


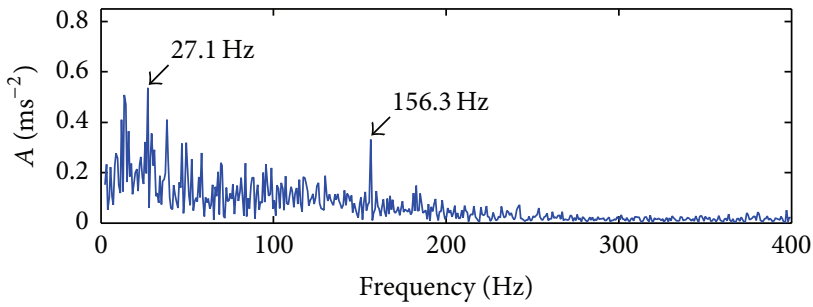

(a)

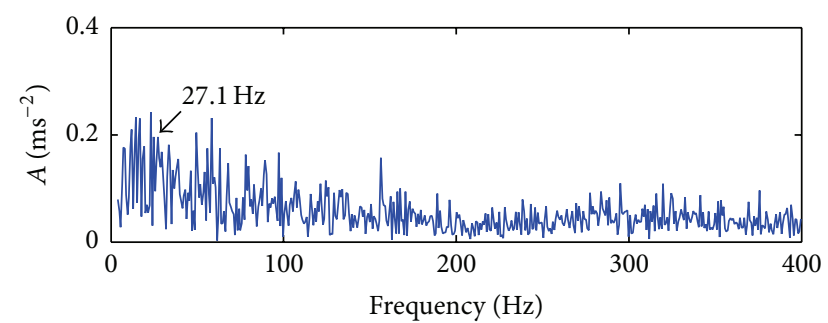

(b)

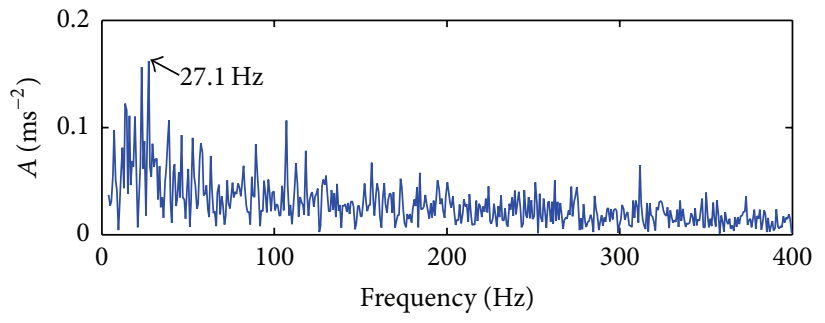

(c)

FigURE 19: Envelope spectra of the reconstructed component signals (crack width of $1 \mathrm{~mm}$ ). (a) First peak. (b) Third peak. (c) Fourth peak.

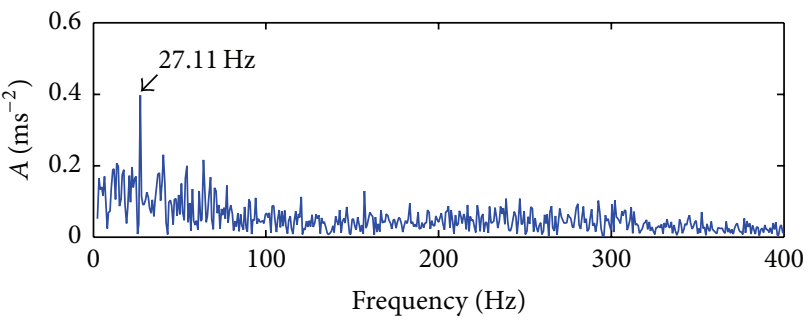

(a)

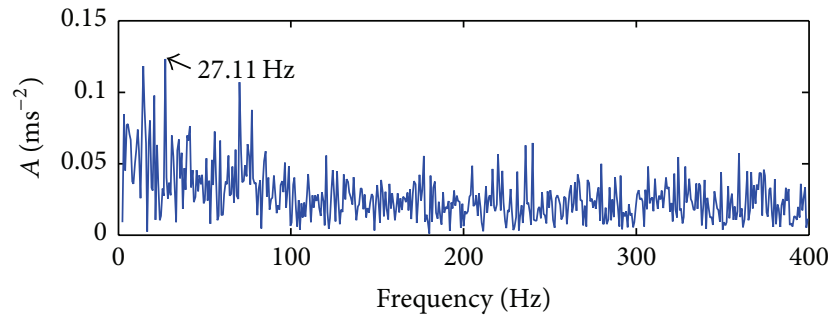

(b)

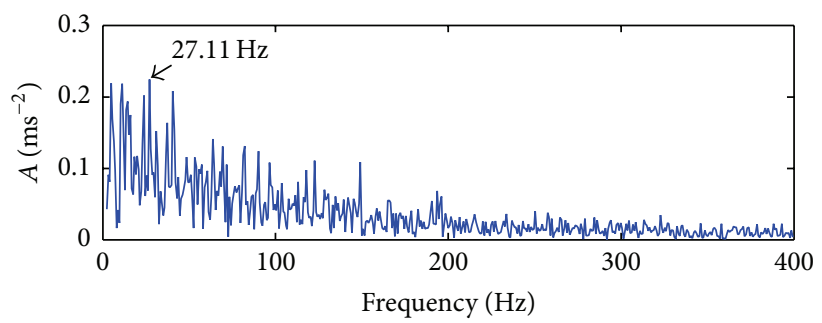

(c)

FIGURE 20: Envelope spectra of the reconstructed component signals (crack width of $0.8 \mathrm{~mm}$ ). (a) First peak. (b) Second peak. (c) Third peak.

\section{Acknowledgments}

This research is supported by the National Natural Science Foundation of China (Grant 11572167). The authors would like to express gratitude to their lab associate Xiang-yi Dang who built the rolling bearing test rig. The authors would like to also express their special thanks to the editors and reviewers for their detailed review work.

\section{References}

[1] G. Chen, T.-F. Hao, X.-Y. Cheng, B. Zhao, and H.-F. Wang, "Sensitivity analysis of fault diagnosis of aero-engine rolling bearing based on vibration signal measured on casing," Journal of Aerospace Power, vol. 29, no. 12, pp. 2874-2884, 2014.

[2] D. N. Brown, "Envelope analysis detects bearing faults before major damage occurs," Pulp and Paper, vol. 63, no. 13, pp. 113117, 1989.

[3] W. Du, Z. Wang, X. Gong, L. Wang, and G. Luo, "Optimum IMFs selection based envelope analysis of bearing fault diagnosis in plunger pump," Shock and Vibration, vol. 2016, Article ID 1248626, 8 pages, 2016

[4] M. Kang, J. Kim, B. K. Choi, and J. M. Kim, "Envelope analysis with a genetic algorithm-based adaptive filter bank for bearing fault detection," The Journal of the Acoustical Society of America, vol. 138, no. 1, pp. EL65-EL70, 2015. 
[5] X. Q. Wang, Y. F. Li, and T. Rui, "Bearing diagnosis method based on Hilbert and deep belief network," Journal of Vibroengineering, vol. 17, no. 3, pp. 1295-1308, 2015.

[6] N. E. Huang, Z. Shen, S. R. Long et al., "The empirical mode decomposition and the Hilbert spectrum for nonlinear and non-stationary time series analysis," Proceedings of the Royal Society of London A: Mathematical, Physical and Engineering Sciences, vol. 454, no. 1971, pp. 903-995, 1998.

[7] Z. Wu and N. E. Huang, "A study of the characteristics of white noise using the empirical mode decomposition method," Proceedings of the Royal Society A: Mathematical, Physical and Engineering Sciences, vol. 460, no. 2046, pp. 1597-1611, 2004.

[8] P. Flandrin, G. Rilling, and P. Gonçalvés, "Empirical mode decomposition as a filter bank," IEEE Signal Processing Letters, vol. 11, no. 2, pp. 112-114, 2004.

[9] J. S. Smith, "The local mean decomposition and its application to EEG perception data," Journal of the Royal Society Interface, vol. 2, no. 5, pp. 443-454, 2005.

[10] K. Dragomiretskiy and D. Zosso, "Variational mode decomposition," IEEE Transactions on Signal Processing, vol. 62, no. 3, pp. 531-544, 2014.

[11] K. H. Zhu, X. G. Song, and D. X. Xue, "Incipient fault diagnosis of roller bearings using empirical mode decomposition and correlation coefficient," Journal of Vibroengineering, vol. 15, no. 2, pp. 597-603, 2013.

[12] M. Han and J. Pan, "A fault diagnosis method combined with LMD, sample entropy and energy ratio for roller bearings," Measurement, vol. 76, pp. 7-19, 2015.

[13] X. Zhang, J. Kang, L. Xiao, J. Zhao, and H. Teng, "A new improved Kurtogram and its application to bearing fault diagnosis," Shock and Vibration, vol. 2015, Article ID 385412, 22 pages, 2015.

[14] Y.-G. Leng, A.-Z. Zheng, and S.-B. Fan, "SVD componentenvelope detection method and its application in the incipient fault diagnosis of rolling bearing," Journal of Vibration Engineering, vol. 27, no. 5, pp. 794-800, 2014.

[15] Y.-X. Zhang, X.-L. Wang, S. Zhang, and J.-P. Zhu, "Rolling element bearing fault diagnosis based on singular value decomposition and correlated kurtosis," Journal of Vibration and Shock, vol. 33, no. 11, pp. 167-171, 2014.

[16] R. Golafshan and K. Y. Sanliturk, "SVD and Hankel matrix based de-noising approach for ball bearing fault detection and its assessment using artificial faults," Mechanical Systems and Signal Processing, vol. 70-71, pp. 36-50, 2016.

[17] Z. R. Pan and Z. J. Qiao, "Feature extraction based on improved SVD denoising and spectral kurtosis in early fault diagnosis of rolling element bearings," in Proceedings of the 5th International Symposium on Test Automation \& Instrumentation, vol. 2014, pp. 14-21, 2014.

[18] Y. Tian, J. Ma, C. Lu, and Z. Wang, "Rolling bearing fault diagnosis under variable conditions using LMD-SVD and extreme learning machine," Mechanism and Machine Theory, vol. 90, pp. 175-186, 2015.

[19] H. Jiang, J. Chen, G. Dong, T. Liu, and G. Chen, "Study on Hankel matrix-based SVD and its application in rolling element bearing fault diagnosis," Mechanical Systems and Signal Processing, vol. 52-53, no. 1, pp. 338-359, 2015.

[20] Z. Wang, C. Lu, Z. Wang, H. Liu, and H. Fan, "Fault diagnosis and health assessment for bearings using the MahalanobisTaguchi system based on EMD-SVD," Transactions of the Institute of Measurement and Control, vol. 35, no. 6, pp. 798-807, 2013.
[21] Z. J. Qiao and Z. R. Pan, "SVD principle analysis and fault diagnosis for bearings based on the correlation coefficient," Measurement Science and Technology, vol. 26, no. 8, Article ID 085014, 2015.

[22] B. Muruganatham, M. A. Sanjith, B. Krishnakumar, and S. A. V. Satya Murty, "Roller element bearing fault diagnosis using singular spectrum analysis," Mechanical Systems and Signal Processing, vol. 35, no. 1-2, pp. 150-166, 2013.

[23] X. Zhao and B. Ye, "Selection of effective singular values using difference spectrum and its application to fault diagnosis of headstock," Mechanical Systems and Signal Processing, vol. 25, no. 5, pp. 1617-1631, 2011.

[24] X. Zhao, B. Ye, and T. Chen, "Extraction method of faint fault feature based on wavelet-SVD difference spectrum," Journal of Mechanical Engineering, vol. 48, no. 7, pp. 37-48, 2012.

[25] P. P. Kanjilal and S. Palit, "On multiple pattern extraction using singular value decomposition," IEEE Transactions on Signal Processing, vol. 43, no. 6, pp. 1536-1540, 1995.

[26] P. P. Kanjilal and G. Saha, "Fetal ECG extraction from single channel maternal ECG using SVD and SVR spectrum," in Proceedings of the IEEE 17th Annual Conference Engineering in Medicine and Biology Society, vol. 1, pp. 187-188, IEEE, Montreal, Canada, September 1997.

[27] X.-Z. Zhao, B.-Y. Ye, and T.-J. Chen, "Influence of matrix creation way on signal processing effect of singular value decomposition," Journal of South China University of Technology (Natural Science), vol. 36, no. 9, pp. 86-93, 2008.

[28] G. L. McDonald, Q. Zhao, and M. J. Zuo, "Maximum correlated Kurtosis deconvolution and application on gear tooth chip fault detection," Mechanical Systems and Signal Processing, vol. 33, pp. 237-255, 2012. 


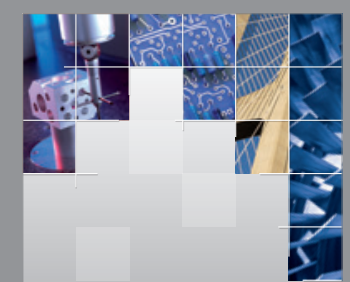

\section{Enfincering}
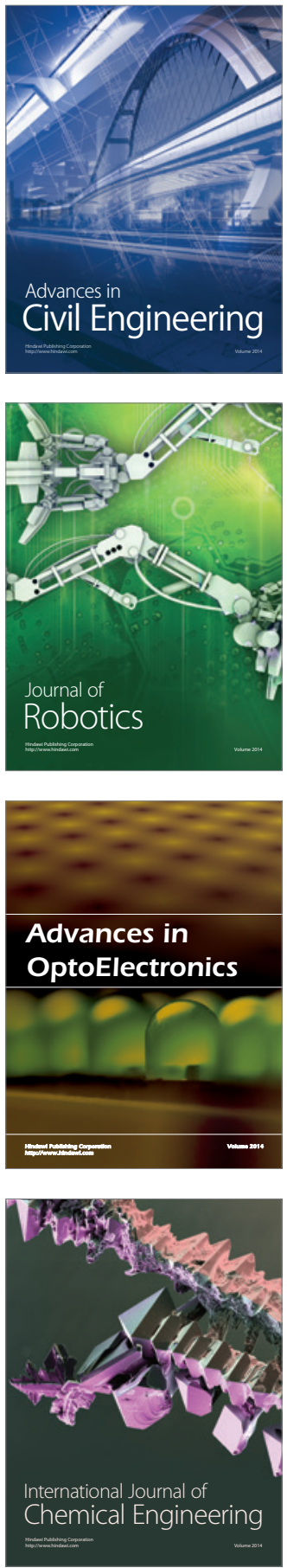

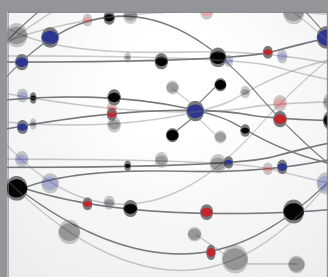

The Scientific World Journal

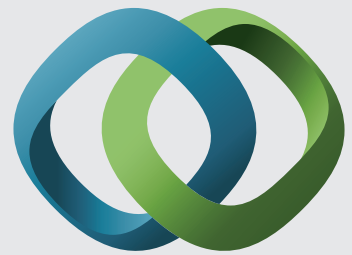

\section{Hindawi}

Submit your manuscripts at

http://www.hindawi.com
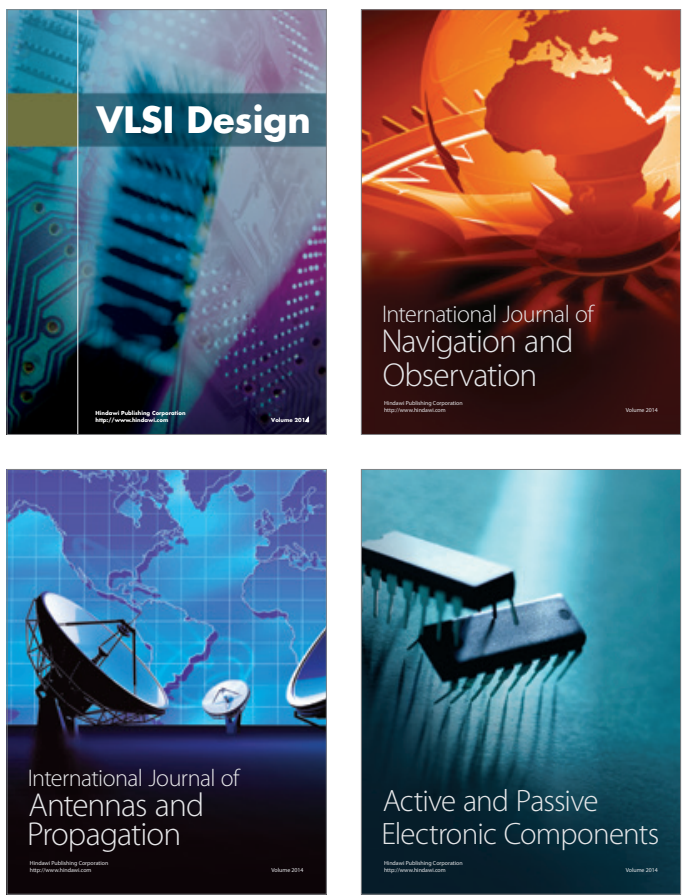
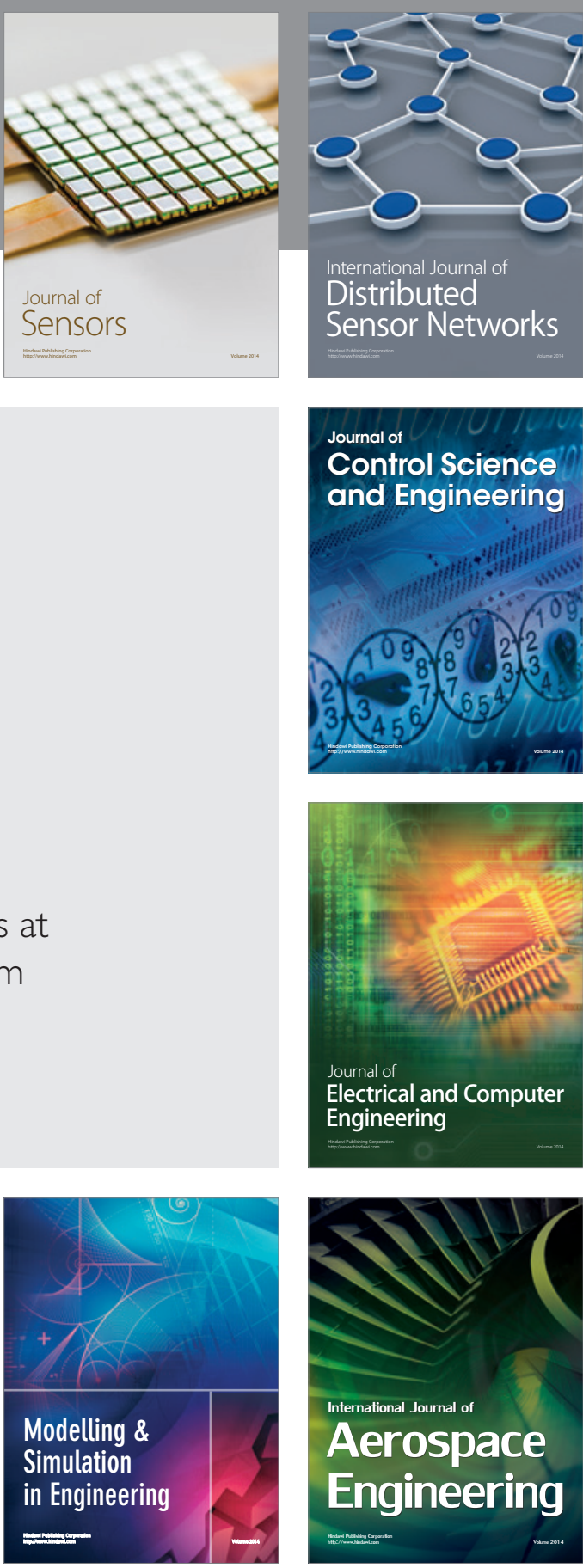

International Journal of

Distributed

Sensor Networks

Journal of

Control Science

and Engineering
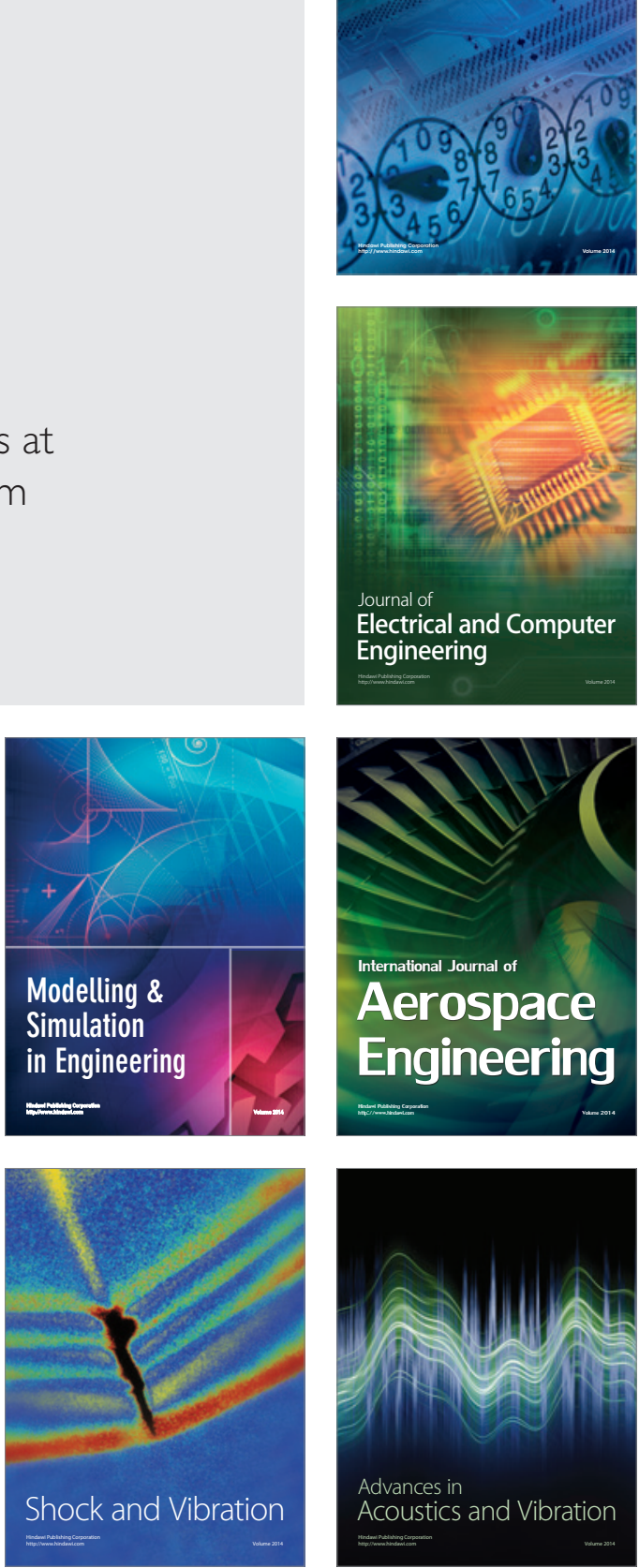